\title{
The First Galaxies and the Likely Discovery of Their Fossils in the Local Group
}

\author{
Massimo Ricotti \\ Department of Astronomy, University of Maryland, College Park, MD 20742, USA \\ Correspondence should be addressed to Massimo Ricotti, ricotti@astro.umd.edu \\ Received 22 May 2009; Revised 17 September 2009; Accepted 5 November 2009 \\ Academic Editor: Andrey V. Kravtsov
}

Copyright () 2010 Massimo Ricotti. This is an open access article distributed under the Creative Commons Attribution License, which permits unrestricted use, distribution, and reproduction in any medium, provided the original work is properly cited.

The lower bound for the mass of a galaxy is unknown, as are the typical luminosity of the smallest galaxies and their numbers. The answers depend on the extent to which star formation in the first population of small mass halos may be suppressed by radiative feedback loops. If early populations of dwarf galaxies did form in significant number before reionization, their "fossils" should be found today in the Local Group. This paper reviews our ongoing efforts to simulate and identify fossil dwarfs in the Local Group. It is widely believed that reionization stopped star formation in fossil dwarfs. However, here we dispute this idea and discuss a physical mechanism whereby recent episodes of star formation would be produced in some fossil dwarfs that, hence, may characterized by a bimodal star formation history. The same mechanism could turn dark halos that failed to form stars before reionization into gas-rich "dark galaxies". We believe that current observational data supports the thesis that a fraction of the new ultra-faint dwarfs discovered in the Local Group are fossil dwarfs and we predict the existence of a population of ultra-faint dwarfs with lower surface brightness than currently observed.

\section{Introduction}

There are many questions that remain open in cosmology with regard to the mass, number, and properties of the smallest galaxies in the universe. Have we already discovered the smallest galaxies in the universe or are we still missing an elusive but large population of ultrafaint dwarf galaxies?

In cold dark matter (CDM) cosmologies most of the dark halos that formed before reionization had masses smaller than $10^{8}-10^{9} \mathrm{M}_{\odot}$ (e.g., [1]). The small mass halos that survived tidal destruction to the modern epoch, were they able to form stars, would constitute a subpopulation of dwarf satellites orbiting larger halos. Small mass dark halos significantly outnumber more massive galaxies like the Milky Way and can be located in the voids between luminous galaxies (e.g., $[2,3])$.

However, until recently (i.e., before 2005) observations did not show a large number of satellites around massive galaxies like the Milky Way and Andromeda. This became known as the "missing galactic satellite problem" $[4,5]$. The voids between bright galaxies appear to be devoid of dwarf galaxies [6-8]. While the abundance of dwarfs in large voids may not pose a problem to CDM cosmology, as shown by Tinker and Conroy [9], it is unclear whether the predictions of the number of faint dwarfs in the Local Group are consistent with both the number of observed Milky Way dwarf satellites and the number of relatively isolated dwarfs in the local voids.

Historically, the discrepancy between observation and theory on the number of dwarf galaxies has been interpreted in two ways: (1) as a problem with the CDM paradigm that could be solved by a modification of the dark matter properties-for instance, by introducing warm dark matter (e.g., [10]) —or (2) as an indicator of feedback processes that are exceptionally efficient in preventing star formation in small mass halos, which remain mostly dark (e.g., [11]).

The recently discovered population of ultrafaint dwarfs [12-22] in combination with a proper treatment of observational incompleteness [23-26] has increased the estimated number of Milky Way satellites to a level that can be more easily reconciled with theoretical expectations. For instance, the suppression of dwarf galaxy formation due to intergalactic medium (IGM) reheating during reionization $[2,3,27-40]$, in conjunction with a strong suppression 
of star formation in small mass pre-reionization dwarfs, may be sufficient to explain the observed number of Milky Way satellites. In the near future we can hope to answer perhaps a more interesting question: what is the minimum mass that a galaxy can have? This is a nontrivial and fundamental question in cosmology. Answering it requires a better understanding of the feedback mechanisms that regulate the formation of the first galaxies before reionization and the details of the process of reionization feedback itself.

The formation of the first dwarf galaxies-before reionization-is self-regulated on cosmological distance scales. This means that the fate of small mass halos (i.e., whether they remain dark or form stars) depends on local and global feedback effects. This type of galaxy feedback differs from the more familiar model operating in normal galaxies (e.g., SN explosions, AGN feedback, etc.), where the feedback is responsible for regulating the star formation rate within the galaxy itself but does not impact star formation in other distant galaxies. Rather, before reionization, each protodwarf galaxy reacts to the existence of all the others. Different theoretical assumptions and models for the cosmological self-regulation mechanisms will, of course, produce different predictions for the number and luminosity of the first dwarf galaxies [11, 41-44].

We now introduce the basic concepts on how feedbackregulated galaxy formation operates in the early universe (i.e., before reionization).

A cooling mechanism for the gas is required in order to initiate star formation in dark halos. In proto-galaxies that form after reionization this is initially provided by hydrogen Lyman-alpha emission. This cooling is efficient at gas temperatures of $20,000 \mathrm{~K}$ but becomes negligible below $T \sim 10,000 \mathrm{~K}$. Later, as the temperature drops below $10,000 \mathrm{~K}$, the cooling is typically provided by metal line cooling. In the first galaxies, however, both these cooling mechanisms may be absent. This is because the first dwarf galaxies differ when compared to present-day galaxies in two respects: (1) they lack important coolants-such as carbon and oxygen, because the gas is nearly primordial in composition, and (2) due to the smaller typical masses of the first dark halos, the gas initially has a temperature that is too low to cool by Lyman-alpha emission.

The gas in small mass halos with circular velocity $v_{\text {vir }}=\left(G \mathrm{M}_{\mathrm{tot}} / r_{\text {vir }}\right)^{1 / 2} \lesssim 20 \mathrm{~km} \mathrm{~s}^{-1}$, where $r_{\text {vir }}$ is the virial radius_-roughly corresponding to a mass $M_{\text {tot }} \lesssim 10^{8} \mathrm{M}_{\odot}$ at the typical redshift of virialization-has a temperature at virialization $T \lesssim 10,000 \mathrm{~K}$. Hence, if the gas has primordial composition, it is unable to cool by Lyman-alpha emission and initiate star formation unless it can form a sufficient amount of primordial $\mathrm{H}_{2}$ (an abundance $x_{\mathrm{H}_{2}} \gtrsim 10^{-4}$ is required). Because molecular hydrogen is easily destroyed by far-ultraviolet (FUV) radiation in the Lyman-Werner bands $(11.3<h v<13.6 \mathrm{eV})$ emitted by the first stars, it is widely believed that the majority of galaxies with $v_{\mathrm{vir}}<$ $20 \mathrm{~km} \mathrm{~s}^{-1}$ remain dark (e.g., [11]). However, several studies show that even if the FUV radiation background is strong, a small amount of $\mathrm{H}_{2}$ can always form, particularly in relatively massive halos with virial temperature of several thousands of degrees $[43,45]$. Thus, negative feedback from
FUV radiation may only delay star formation in the most massive pre-reionization dwarfs rather than fully suppress it $[46,47]$. We will argue later that hydrogen ionizing radiation $(h v>13.6 \mathrm{eV})$ in the extreme-ultraviolet (EUV) plays a far more important role in regulating the formation of the first galaxies than FUV radiation. Thus, in our opinion, models that do not include 3D radiative transfer of $\mathrm{H}$ and He ionizing radiation cannot capture the most relevant feedback mechanism that regulates galaxy formation in the early universe $[41,48]$.

After reionization, the formation of dwarf galaxies with $v_{\text {vir }}<20 \mathrm{~km} \mathrm{~s}^{-1}$ is strongly inhibited by the increase in the Jeans mass in the IGM. Thus, according to this model, reionization feedback and negative feedback due to $\mathrm{H}_{2}$ photodissociation by the FUV background (important before reionization) determine the mass of the smallest galactic building blocks. The resultant circular velocity of the smallest galactic building blocks is $v_{\text {vir }} \sim 20 \mathrm{~km} \mathrm{~s}^{-1}$, roughly corresponding to masses $M_{\text {tot }} \sim 10^{8}-10^{9} \mathrm{M}_{\odot}$. If this is what really happens in the early universe, the "missing Galactic satellite problem" can be considered qualitatively solved because the predicted number of Milky Way satellites with $v_{\text {vir }}>20 \mathrm{~km} \mathrm{~s}^{-1}$ is already comparable to the estimated number of observed satellites after applying completeness corrections (although this model may still have problems reproducing the observations in detail).

However, as briefly mentioned above, we have argued for some time that most simulations of the first galaxies cannot capture the main feedback mechanism operating in the early universe because they do not include a key physical ingredient: radiative transfer of $\mathrm{H}$ and $\mathrm{He}$ ionizing radiation. Our simulations of the formation of the first galaxies are to date the only simulations of a cosmologically representative volume of the universe (at $z \sim 10$ ) that include 3D radiative transfer of $\mathrm{H}$ and $\mathrm{He}$ ionizing radiation $[41,44,48]$. Figure 1 shows the evolution of ionized bubbles around the first galaxies in a cubic volume of $1.5 \mathrm{Mpc}$ in size at redshifts $z=$ $21.2,17.2,15.7,13.3$ from one of our simulations. The results suggest that negative feedback from the FUV background is not the dominant feedback mechanism that regulates galaxy formation before reionization. Rather, "positive feedback" on $\mathrm{H}_{2}$ formation from ionizing radiation [30,49] dominates over the negative feedback of $\mathrm{H}_{2}$ dissociating radiation. Hence, a strong suppression of galaxy formation in halos with $v_{\mathrm{vir}}<20 \mathrm{~km} \mathrm{~s}^{-1}$ does not take place. In this latter case, some galactic satellites would be the fossil remnants of the first galaxies. Comparisons of simulated pre-reionization fossils to dwarf spheroidals in the Local Group show remarkable agreement in properties (see [50], hereafter RG05). Based on the results of the simulations, we also suggested the existence of the ultrafaint population before it was discovered about a year later (see RG05 [51]).

1.1. Definition of "Pre-Reionization Fossils". Throughout this paper we define "pre-reionization fossils" as the dwarfs hosted in halos with a maximum circular velocity remaining below $20 \mathrm{~km} \mathrm{~s}^{-1}$ at all times during their evolution: $v_{\max }(t)<$ $20 \mathrm{~km} \mathrm{~s}^{-1}$. It will become clear in this paper that this definition is not directly related to the ability of fossils to 


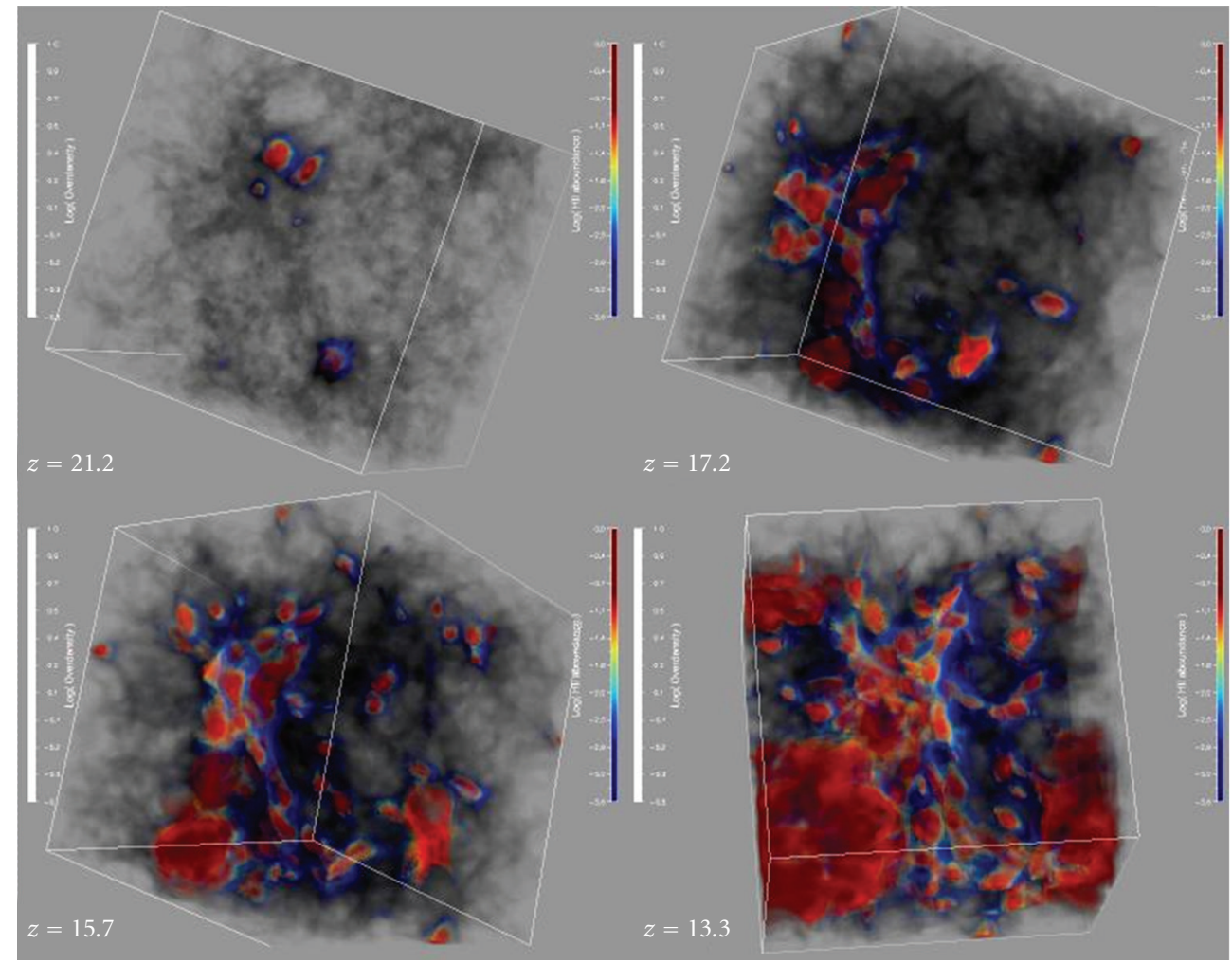

FIGURE 1: 3D rendering of cosmological H II regions (fully ionized gas is red and partially ionized gas is blue) around the first galaxies in a box of 1.5 Mpc. The four boxes show a time sequence at redshifts $z=21.2,17.2,15.7,13.3$ for the simulation S2 from Ricotti et al. [41]. The rendering shows several tens of small size H II regions around the first galaxies (there are a few hundreds of galaxies in this volume). A movie of the same simulation shows that the H II regions are short lived: they form and expand to a size comparable to the large-scale filamentary structure of the IGM and recombine, promoting the formation of molecular hydrogen inside the relic $\mathrm{H}$ II regions.

retain gas and form stars after reionization: in Section 4 we describe a mechanism in which small mass halos with $v_{\max }(t)<20 \mathrm{~km} \mathrm{~s}^{-1}$ are able to have a late phase of gas accretion and possibly star formation.

Our definition of fossil reflects the special cooling mechanisms and feedback processes that regulate star formation and the number of luminous halos with $v_{\max }(t)<20 \mathrm{~km} \mathrm{~s}^{-1}$, before and after reionization. In protofossil galaxies-even adopting the most conservative assumption of maximum efficiency of shock heating of the gas during virializationthe gas is heated to a temperature below $T \sim 10,000 \mathrm{~K}$. Thus the gas cannot cool by Lyman-alpha emission, a very efficient coolant. The cooling of the gas is dominated either by $\mathrm{H}_{2}$ roto-vibrational line emission or by metal cooling, important if the metallicity exceeds $Z \sim 10^{-3} Z_{\odot}$ (e.g., [44, 52-54]). These coolants are much less efficient than Lyman-alpha emission. Moreover, $\mathrm{H}_{2}$ abundance and cooling is modulated and often suppressed by the FUV and EUV radiation fields. The FUV radiation in the $\mathrm{H}_{2}$ Lyman-Werner bands and hard ultraviolet radiation have large mean free paths with respect to the typical distances between galaxies; thus their feedback is global in nature. Qualitatively, this explains why the first galaxies have low luminosities and low surface-brightness, similar to dwarf spheroidal (dSph) galaxies in the Local Group (see RG05 [51, 55]).
Simulations also show that stars in the the first galaxies do not form in a disk but in a spheroid (see RG05 [44]). A thin galactic disk is not formed because of the high merger rates and the low masses of dark halos in the early universe. Roughly, pre-reionization fossils have a mass at virialization $M_{\text {tot }}<10^{8} \mathrm{M}_{\odot}$, assuming that they form at $z_{\mathrm{vir}} \sim 10$, but their mass may increase by up to one order of magnitude by $z=0$ due to secondary infall (see RGS02a, b [44]). Secondary infall does not affect $v_{\max }$, which remains roughly constant after virialization.

1.2. Pre-Reionization Fossils and Reionization. The critical value of $v_{\text {max,crit }}$ for which dwarf galaxy formation is suppressed by reionization feedback is close to the $20 \mathrm{~km} \mathrm{~s}^{-1}$ value that defines a fossil, but it is not necessarily the same value. Indeed, it can be significantly larger than $20 \mathrm{~km} \mathrm{~s}^{-1}$ if the IGM is heated to $T \gg 10,000 \mathrm{~K}$ [56]. Thus, we expect that the virialization of new "pre-reionization fossils" is strongly suppressed after reionization due to IGM reheating (i.e., they mostly form before reionization). However, prereionization fossils and dark halos with $v_{\max }<20 \mathrm{~km} \mathrm{~s}^{-1}$ that virialized before reionization may accrete gas and, in certain cases, form new stars after reionization at redshifts $z<1-2$ [3]. 
Unfortunately, the value of $v_{\max , \text { crit }}$ is uncertain due to our poor understanding of the thermal history of the IGM [56]. The uncertainty surrounding the IGM equation of state may partially explain the differences found in literature on the values for $v_{\max , c r i t}$ and the different levels of suppression of star formation as a function of the halo mass after reionization (e.g., $[2,33,36,40])$. Regardless of assumptions for the reionization feedback model, one should bear in mind that no halo with $v_{\max }<20 \mathrm{~km} \mathrm{~s}^{-1}$ can form stars after reionization unless the gas in those halos has been significantly pre-enriched with metals. For instance, the model by Koposov et al. [57] assumes star formation after reionization in halos as small as $v_{\max } \sim 10 \mathrm{~km} \mathrm{~s}^{-1}$. With this assumption they find that their model is consistent with observations of ultrafaint dwarfs but claim that fossils are not needed to explain the data. However, star formation in such small halos can only take place in a gas that was pre-enriched with metals, suggesting the existence of older populations of stars in those halos. Indeed, according to our definition, the smallest post-reionization dwarfs with $10 \mathrm{~km} \mathrm{~s}^{-1}<v_{\max }<$ $20 \mathrm{~km} \mathrm{~s}^{-1}$ in the Koposov et al. [57] model are "fossils." As stated above, fossils may also be able to form stars after reionization due to a late phase of cold gas accretion from the IGM [3].

\subsection{Identification of Pre-Reionization Fossils in Observations.} Pre-reionization fossils are not easily identifiable because $v_{\max }$ cannot be measured directly from observations. Understanding the star formation history of dwarf galaxies may help in this respect, as fossils likely show some degree of suppression of their star formation rate occurring about $12.5 \mathrm{Gyr}$ ago due to reionization. However, their identification based on their star formation history may be complicated because some pre-reionization fossils in the last 10 Gyr may have had a late phase of gas accretion and star formation. The caveat is that star formation histories cannot be measured with accuracy better than to within 1-2 Gyr and the accuracy becomes increasingly poorer for old stellar populations. Thus, it is impossible to prove whether an old population of stars formed before reionization (which happened about 1 Gyr after the Big Bang) or at $z \sim 3$, when the Milky Way was assembled. Nevertheless, ultrafaint dwarfs that show some degree of bimodality in their star formation history are candidates for being pre-reionization fossils.

According to results by RG05 and Bovill and Ricotti [51], Willman 1, Bootes II, Segue 1, and Segue 2 do not lie on the luminosity-surface brightness relationship of simulated prereionization fossils. This result is based on the assumption that fossil properties are not modified by tides. Their surface brightness is larger than the model predictions for objects with such low-luminosity. Yet undiscovered population of ultrafaints with lower surface brightness is instead predicted by our simulations. It is likely that the properties of the lowest luminosity ultrafaints may have been modified by tidal forces due to their proximity to the Milky Way disk.

Although it is difficult to identify individual fossils, statistical arguments suggest that at least some ultrafaint dwarf galaxies are pre-reionization fossils. This is because the number of satellites from N-body simulations with $v_{\max }(t)>20 \mathrm{kms}^{-1}$ is substantially smaller than the estimated number of observed satellites after completeness corrections. Admittedly the current theoretical and observational uncertainties on the number of satellites are still large. However, if the estimated number (after completeness corrections) of ultrafaint dwarfs increases further, the existence of pre-reionization fossils will be inescapably proven. This is especially the case if a population of ultrafaint dwarfs with luminosities similar to Willman 1, Bootes II, Segue 1, and Segue 2 but surface brightness below the current sensitivity limit of the SDSS - as predicted by our simulations-is discovered.

The possibility of identifying the fossils of the first galaxies in our own backyard is very exciting. It would greatly improve our understanding of the physics involved in self-regulating the formation of the first galaxies before reionization. Clearly, even the launch of the James Webb Space Telescope (JWST) would not yield the wealth of observational data on the formation of the first galaxies that could be obtained by studying ultrafaint galaxies in the Local Group.

The rest of the paper is organized as follows. In Section 2 we briefly review and discuss observational data on Galactic satellites; in Section 3 we summarize the results of simulations of the formation of the first galaxies in a cosmological volume and the effect of reionization feedback on galaxy formation. In Section 4 we discuss a recently proposed model for "late gas accretion" from the IGM onto small mass halos. In Section 5 we compare the theoretical properties of simulated pre-reionization fossils to observations. In Section 6 we compare different ideas for the origin of classical and ultrafaint dwarf spheroidals. We present our conclusions in Section 7.

\section{Observations}

2.1. The Ultrafaint Satellites of the Milky Way and Andromeda. Prior to 2005, the number of observed dwarf satellites of the Milky Way and Andromeda was about 30 [58]. One of the most evident properties of the dwarfs in the Local Group is a type segregation, with "gas free" dwarf spheroidal (dSph) galaxies distributed near the center of their host galaxy and gas rich dwarf Irregulars (dIrr) at larger distances from the galactic centers. Notable exceptions are the Magellanic Clouds that are dIrr less than $100 \mathrm{kpc}$ from the center of the Milky Way and a few isolated dSphs like Tucana and Antlia. One popular explanation for this segregation is the transformation of dIrr into $\mathrm{dSph}$ due to tidal and ram pressure stripping as dwarfs fall toward the Milky Way center $[59,60]$. In addition, simulations showed that the number of dark matter satellites of the Milky Way with mass $>10^{8} \mathrm{M}_{\odot}$ (i.e., with mass sufficiently large to expect star formation in them) was an order of magnitude larger than the number of known dwarf satellites $[4,5]$. This posed a problem for CDM cosmologies.

Since 2005-2006 the number of known Local Group satellites has begun to increase dramatically, with the discovery of a new population of ultrafaint dwarfs. The new galaxies have been discovered by data mining the SDSS and other 
surveys of the halo around M31, resulting in the discovery of 14 new ultrafaint Milky Way satellites [12-19, 24, 61] and 11 new companions for M31 [20-22, 62]. Unofficial reports from members of the SDSS collaboration state that there are actually at least 17 new ultrafaint Milky Way dwarfs, but several of them are as yet unpublished (anonymous referee's private communication).

The new Milky Way satellites have been slowly discovered since SDSS Data Release 2, with the most recent Segue 2 discovered in Data release 7 [63, 64]. Koposov et al. [24] and Walsh et al. [61] systematically searched Data Releases 5 and 6 , respectively. Due to the partial sky coverage of the SDSS, and assuming isotropic distribution of satellites (but see $[65,66])$, the total number of ultrafaint dwarfs in the Milky Way should be at least 5.15 times larger than the observed number [25]. With this simple but conservative correction, the number of Milky Way satellites within $400 \mathrm{kpc}$ is about $12+5.15 \times(14 \pm 3.7) \sim 84 \pm 19$. The quoted uncertainty is simply Poisson error due to the relatively small number of known ultrafaint dwarfs.

In estimating the completeness correction for the number of Milky Way dwarfs, one should account for selection effects inherent in the method used to find the ultrafaints in the SDSS data. In addition to completeness corrections for the survey's coverage of the sky, the most important selection effect is the total number of stars from the object seen in the survey, that is, horizontal branch stars or main sequence and/or red giant stars for the lowest luminosity ultrafaints like Coma or Segue 1. This sets a limiting surface brightness cutoff at roughly $30 \mathrm{mag} \operatorname{arcsec}^{-2}$ for the SDSS [24] (but see Martin et al. [67] that find a limiting surface brightness about 6.4 times larger). There is also a distancedependent absolute magnitude cutoff. The efficiency of finding ultrafaint dwarfs by data mining the SDSS typically drops rapidly at Galactocentric distances beyond 50-150 kpc for the ultrafaints (depending on their luminosity) [23$25,61]$. Of the new Milky Way dwarfs, only Leo $\mathrm{T}$ is well beyond this distance threshold, and 11 of the 14 new Milky Way satellites are within $200 \mathrm{kpc}$. (Leo T was found because it contains a young stellar population and gas. Otherwise, it would not have been identified as an ultrafaint dwarf due to its large Galactocentric distance.)

The luminous satellites can be radially biased. So, the abundance of the faintest satellites within $50 \mathrm{kpc}$, that is the most complete sample, may not be used to correct for incompleteness at larger distances from the Galactic center without prior knowledge of this bias. And, of course, satellites of different luminosity and surface brightness will have different completeness limits. These selection biases have been considered in a paper by Tollerud et al. [25]. Their study finds that there may be between 300 to 600 luminous satellites within $400 \mathrm{kpc}$. Their estimate for the number of luminous satellites within a Galactocentric distance of $200 \mathrm{kpc}$ is between 176 to 330 .

Recent surveys of M31 [20, 22, 62] have covered approximately a quarter of the space around the M31 spiral. The surveys have found 11 new M31 satellites, bringing the total number to 19. If we make a simple correction for the covered area of the survey, the estimated number of
M31 satellites, including the new dwarfs, increases from 8 to $52 \pm 13$.

The sensitivity limits of the surveys for Andromeda do not allow the detection of ultrafaint dwarfs that would correspond with those with the lowest luminosity found in the Milky Way. However, despite the fact that Andromeda and the Milky Way are thought to have approximately the same mass (within a factor of two), their satellite systems show interesting differences for even the satellites at the brighter end of the luminosity function. For instance, there are small differences in the galactocentric distance distribution of satellites and in the morphology of the satellites (e.g., number of dIrr, dE, and dSphs).

2.2. Peculiar Ultrafaint Dwarfs. Many of the newly discovered dwarfs are dSphs with a dominant old population of stars and virtually no gas, which makes them candidates for being pre-reionization fossils. However, there are notable exceptions that we discuss below that may not perfectly fit the properties of simulated "fossils." For instance, the dwarf galaxy Leo $\mathrm{T}$ resembles all the other ultrafaints but has gas and recent star formation $[14,23]$. We argue that Leo $\mathrm{T}$ could be a true "fossil" with $v_{\max }(t)<20 \mathrm{~km} \mathrm{~s}^{-1}$ but may have experienced a late phase of gas accretion and star formation due to the mechanism discussed in Section 4.

Leo $\mathrm{T}$ has a stellar velocity dispersion of $\sigma_{\text {LeoT }}=7.5 \pm$ $1.6 \mathrm{~km} \mathrm{~s}^{-1}$ [23] or an estimated dynamical mass of $10^{7} \mathrm{M}_{\odot}$ within the stellar spheroid (although its total halo mass may be much larger). Leo $\mathrm{T}$ shows no sign of recent tidal destruction by either the Milky Way or M31 [68] and is located in the outskirts of the Milky Way at a Galactocentric distance of $400 \mathrm{kpc}$. Leo T photometric properties are consistent with those of pre-reionization fossils. On the other hand, the halo of Leo T could be sufficiently massive $\left(v_{\max }(t)>20 \mathrm{~km} \mathrm{~s}^{-1}\right)$ to retain or accrete mass after reionization and not be a prereionization fossil. However, as discussed in Section 4 it is also possible that Leo $\mathrm{T}$ is a pre-reionization fossil that has been able to accrete gas from the IGM at late times due to an increase in the concentration of its dark halo and a decrease in the IGM temperature [3]. Under this scenario, Leo T stopped forming stars after reionization but was able to start accreting gas again from the IGM at $z \lesssim 1-2$ and therefore would have a bimodal stellar population. De Jong et al. [68] have found evidence for bimodal star formation in Leo $\mathrm{T}$. Our model would explain why Leo $\mathrm{T}$ does not resemble other dIrr and is similar to dSphs and pre-reionization fossils, while not suffering significant tidal stripping.

Willman 1, Bootes II, Segue1, and Segue2 are among the lowest luminosity ultrafaint dwarfs discovered so far; however, they do not fit the typical properties of prereionization fossils (see Section 5). For instance, Willman 1 has a dynamical mass within the largest stellar orbit $(r \sim 100 \mathrm{pc})$ of $5 \times 10^{5} \mathrm{M}_{\odot}$ and a mass-to-light ratio $\sim 470$, similar to other ultrafaint dwarfs [17]. However, given its low luminosity, Willman 1 has central surface brightness that is too large when compared to simulated "fossils." Simulated fossils with luminosities $L_{\mathrm{V}}<10^{3} \mathrm{~L}_{\odot}$ should have a typical surface brightness that falls below the detection limit of $\sim 30 \mathrm{mag} \mathrm{arcsec}^{-2}$ estimated for the SDSS [24]. Hence, 
the lowest luminosity fossils may still be undiscovered. Although the nature of the lowest luminosity ultrafaints is unknown, due to selection effects they can only be found within $\sim 50 \mathrm{kpc}$ of the Galactic center. Thus it is possible, and perhaps to be expected, that their properties have been affected by tidal forces $[60,69]$.

\section{Formation of First Galaxies in CDM}

The first episodes of star formation in the universe are thought to take place at redshift $z \sim 30-50$, in the center of dark matter halos with typical mass $M_{\text {tot }} \sim 10^{5}-10^{6} \mathrm{M}_{\odot}$. The gas in these halos is metal free and simulations show that a single or binary massive star per halo is formed [7077]. Such stars are called Pop III and their mass, although not well constrained by the simulations, is quite large: in the range between $20 \mathrm{M}_{\odot}$ and a few $100 \mathrm{M}_{\odot}$. Whether or not we can refer to minihalos containing a single massive star (or a binary star) in their center as the "first galaxies" is debatable. However, the crucial point to be made here is that there is a gap of 2 to 3 orders of magnitude between the typical halo mass in which Pop III stars are born $\left(10^{5}-10^{6} \mathrm{M}_{\odot}\right)$ and the typical mass of the population of dwarf galaxies that are not strongly influenced by radiative and reionization feedback $\left(10^{8}-10^{9} \mathrm{M}_{\odot}\right)$. The primordial dwarfs that fill the gap are those that we refer to as pre-reionization fossils.

If the formation of pre-reionization fossils is not drastically suppressed by radiative feedback, their number may be several orders of magnitude larger than the number of more massive dwarfs. This is because in CDM cosmologies the number of dark halos per unit comoving volume roughly scales with the mass as $N \propto M_{\mathrm{dm}}^{-2}$.

It is widely believed that nearly all halos with mass $M_{\text {tot }}>$ $10^{8}-10^{9} \mathrm{M}_{\odot}$ host luminous galaxies, although there can be substantial disagreement among theorists on their luminosity. However, most of the theoretical controversy rests in understanding the fate of the halos with mass between $10^{6}-10^{8} \mathrm{M}_{\odot}$ and the dominant feedback that determines whether they become luminous or remain dark. We will elaborate on this statement in the next sections.

3.1. Radiative Feedback. Simulating the formation of the first stars is a relatively well-defined initial condition problem, given the cosmological parameters. However, these simple initial conditions must soon be modified to take into account the effects of other newborn stars, whose properties are still quite uncertain. The physics becomes more complex as competing feedback effects determine the fate of the first galaxies: radiative feedback regulates the formation and destruction of $\mathrm{H}_{2}$ and metals are injected into the IGM and into protogalaxies.

\subsubsection{Negative Feedback from $\mathrm{H}_{2}$ Photodissociating Radiation.} The net effect of radiative feedback on the global star formation history of the universe before the redshift of reionization is uncertain. An FUV background (at energies between $11.34 \mathrm{eV}$ and $13.6 \mathrm{eV}$ ) destroys $\mathrm{H}_{2}$, the primary coolant at the start of galaxy formation. The FUV radiation emitted by the first few Pop III stars is sufficient to suppress or delay galaxy formation in halos with circular velocities $v_{\text {vir }}<$ $20 \mathrm{~km} \mathrm{~s}^{-1}$ that are too small to cool by Lyman-alpha emission $[11,43,78,79]$. Hence, according to this scenario, most halos with masses $<10^{8}-10^{9} \mathrm{M}_{\odot}$ remain dark. More work is needed to quantify the level of suppression of galaxy formation and examine how these models compare to observations of Milky Way satellites.

Figure 2 illustrates the effect of $\mathrm{H}_{2}$ dissociating radiation on the IGM. The two panels show slices through a simulation in Ricotti et al. [41] at $z=19.44$ and $z=18.5$. The topright tiles in the two panels show $\mathrm{H}_{2}$ abundance. At $z=19.4$, the $\mathrm{H}_{2}$ has its relic abundance everywhere in the IGM except inside the dissociation spheres around the first galaxies, where it is destroyed. At $z=18.5$, the dissociation spheres are still visible, but the UV background starts to dissociate $\mathrm{H}_{2}$ everywhere in the IGM except the denser filaments.

3.1.2. Positive Feedback Regions. Our main criticism for the "negative feedback" model is that it does not take into account the effect of hydrogen ionizing radiation $[49,80]$ that, according to simulations, may indeed play a dominant role in regulating galaxy formation before reionization [41, 48]. Simulations including 3D radiation transfer show that star formation in the first small mass halos is inefficient, partially due to winds produced by internal UV sources. This produces galaxies that are extremely faint and have very low surface brightnesses. However, our simulations show that a large number of ultrafaint dwarfs (a few hundred galaxies per comoving $\mathrm{Mpc}^{3}$ ) form before reionization at $z \sim 7-10$. Hence, according to this model, the Local Group may contain thousands of ultrafaint dwarf galaxies.

Ionizing radiation from the first stars enhances the production of $\mathrm{H}_{2}$ (we refer to this as "positive feedback") by creating free electrons and promoting the formation of $\mathrm{H}^{-}$, the main catalyst for the formation of $\mathrm{H}_{2}$ in a low metallicity gas [30, 49, 81-83]. Ricotti et al. [49] found that shells of $\mathrm{H}_{2}$ can be continuously created in precursors around the Strömgren spheres produced by ionizing sources and, for a bursting mode of star formation, inside recombining $\mathrm{H}$ II regions. We refer to these shells as "positive feedback regions." This is because the catalyst $\mathrm{H}^{-}$, and hence $\mathrm{H}_{2}$, is formed most efficiently in regions where the gas ionization fraction is about $50 \%$. This local "positive feedback" is difficult to incorporate into cosmological simulations because the implementation of spatially inhomogeneous, time-dependent radiative transfer is computationally expensive.

Figure 3 shows "positive feedback regions" in one of our simulations. The figure shows a slice through a simulation at 4 different times (at $z=17.3,12.2,11.3$, and 10.2). We recognize the two main processes that create $\mathrm{H}_{2}$ in the filaments. In the top-left frame at $z=17.3$ we can see a "positive feedback region" as an irregular shell of $\mathrm{H}_{2}$ surrounding the $\mathrm{H}$ II region that is barely intersected by the slice. In the bottom-left frame $(z=11.3)$ two $\mathrm{H}$ II regions are clearly visible. Inside the $\mathrm{H}$ II regions, the $\mathrm{H}_{2}$ is destroyed. In the bottom-right frame $(z=10.2)$ the $\mathrm{H}$ II regions are recombining (demonstrating that the star formation is 


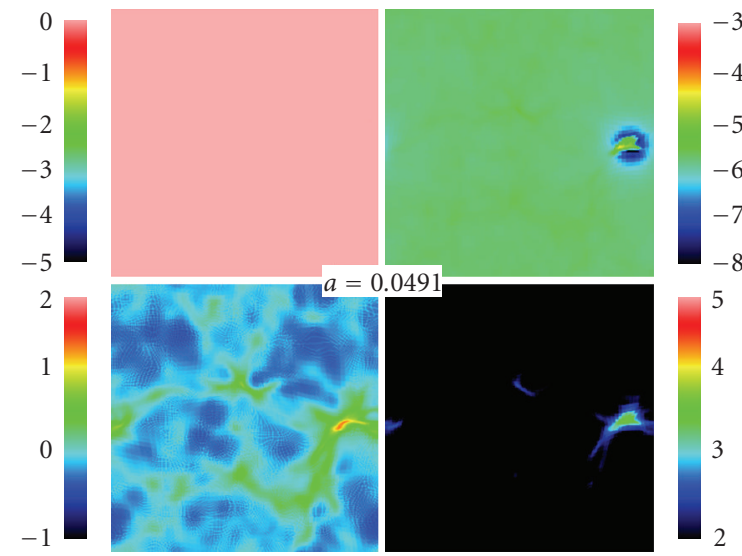

(a)

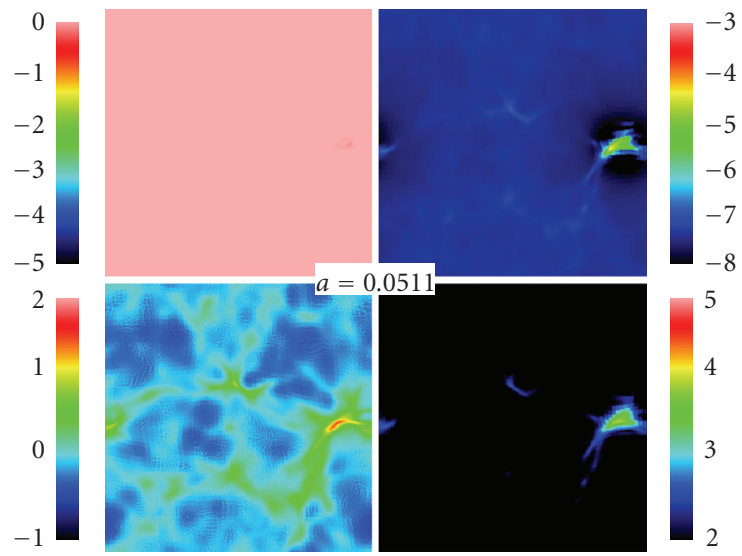

(b)

FIgURE 2: The two $(2 \times 2)$ panels show slices through the most massive object in the simulation 64L05p2 in RGS02b at $z=19.4$ and 18.5 . The box size is $L_{\mathrm{box}}=0.7$ comoving Mpc. Each one of the $2 \times 2$ panels shows in log-scale the neutral hydrogen fraction (top left), the molecular fraction (top right), the gas overdensity (bottom left), and the gas temperature (bottom right). The sequence illustrates the evolution of a $\mathrm{H}_{2}$ dissociation sphere around a single source (panel at $z=19.4$ ) and the dominance of the $\mathrm{H}_{2}$ dissociating background at $z=18.5$.

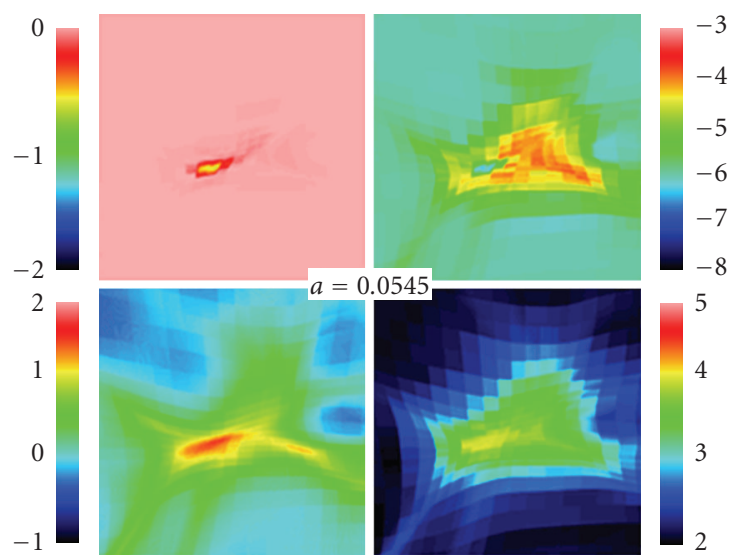

(a)

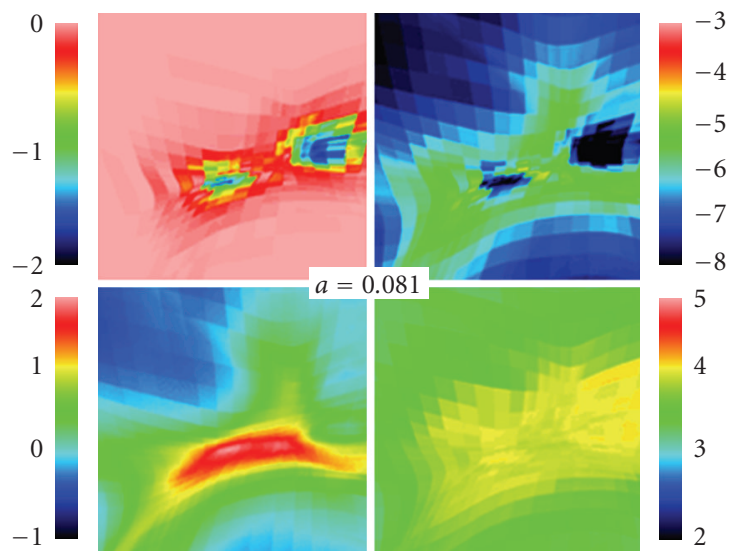

(c)

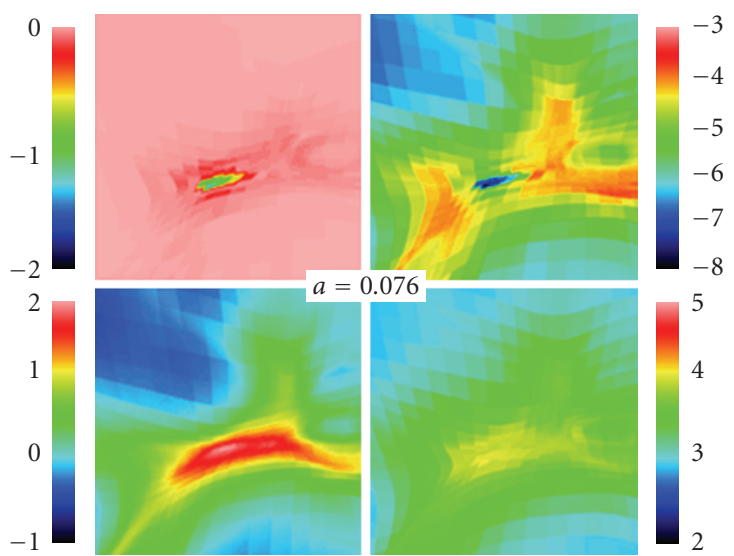

(b)

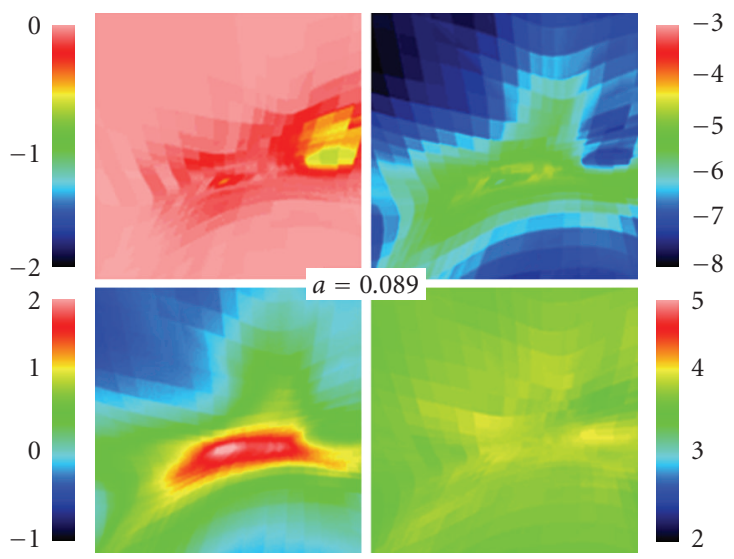

(d)

FIGURE 3: Same as in Figure 2 except for a zoomed region of $0.125^{2} \mathrm{~h}^{-2} \mathrm{Mpc}^{2}$ around the most massive object in the 64L05p3 simulation in RGS02b. In this time sequence of images (top: $z=17.3,12.2$ from left to right; bottom: $z=11.3,10.2$ from left to right) we recognize the two main processes that create $\mathrm{H}_{2}$ in the filaments: "positive feedback regions" in front of $\mathrm{H}$ II regions and the reformation of $\mathrm{H}_{2}$ inside relic $\mathrm{H}$ II regions. The bursting mode of the star formation is evident from the continuous formation and recombination of the $\mathrm{H}$ II regions in the time sequence of the slices. 
bursting) and new $\mathrm{H}_{2}$ is being reformed inside the relic $\mathrm{H}$ II regions. A finer inspection of the time evolution of this slice shows that at least five $\mathrm{H}$ II regions form and recombine between $z=20$ and $z=10$ in this small region of the simulation (Movies of 2D slices and 3D rendering of the simulations are publicly available on the web at the URL: http://www.astro.umd.edu/ ricotti/movies.html).

There are two reasons why our results are still controversial. First, our simulations do not yet have sufficient resolution to ensure their convergence. Second, there are no other published simulations to compare our results with. Only recently have some groups started to include the effect of 3D radiative transfer on hydrodynamics (e.g., $[42,45])$. However, currently there are no other simulations of the formation of the first galaxies in a cosmological volume suited for comparison with observations of dwarfs in the Local Group other than our own $[41,44,48,50$, 51]. Hence, our results may differ from other numerical studies because of the inclusion in the code of the effects of "positive feedback regions" and galactic winds from ionizing radiation.

Simulations by Wise and Abel [42] include a selfconsistent treatment of hydro and $3 \mathrm{D}$ radiative transfer that is more accurate than our approximate, but faster method. However, because the authors use ray-tracing for the radiative transfer, only a few sources of radiation can be simulated at the same time. This limits the volume and number of galaxies that can be simulated. Due to these limitations the simulations are not suited for comparison between the primordial dwarf populations and the ultrafaint dwarfs. In addition, at the moment, the aforementioned simulations do not include metal cooling and the formation of normal stars (other than Pop III).

3.1.3. The Simulations. The simulation used for comparison to observations of ultrafaint dwarfs has been thoroughly described in Ricotti et al. [41, 48] as run "256L1p3." Here we remind the reader that the simulation includes $256^{3}$ dark matter particles, an equal number of baryonic cells, and more than 700,000 stellar particles in a box of size $\sim 1.5$ comoving Mpc. The mass of the dark matter particles in our simulation is $4930 \mathrm{M}_{\odot}$, and real comoving spatial resolution (twice the Plummer softening length) is $150 \mathrm{~h}^{-1} \mathrm{pc}$ (which corresponds to a physical scale of 24 parsecs at $z=8.3$ ). This resolution allows us to resolve cores of all simulated galaxies that would correspond to the observed Local Group dwarfs. The stellar masses are always smaller than the initial baryon mass in each cell but can vary from $\sim 0.6 \mathrm{~h}^{-1} \mathrm{M}_{\odot}$ to $600 \mathrm{~h}^{-1} \mathrm{M}_{\odot}$ with a mean of $6 \mathrm{~h}^{-1} \mathrm{M}_{\odot}$. Stellar particles do not represent individual stars but, in general, a collection of stars (e.g., $\mathrm{OB}$ associations).

The simulation includes most of the relevant physics, including time-dependent spatially-variable radiative transfer using the OTVET approximation [84], detailed radiative transfer in Lyman-Werner bands, and nonequilibrium ionization balance. In addition to primordial chemistry and 3D radiative transfer, the simulations include a subgrid recipe for star formation, metal production by $\mathrm{SNe}$, and metal cooling. The code also includes mechanical feedback by SN explosions. However, we found that for a Salpeter IMF, the effect of SNe is not dominant when compared to feedback produced by ionizing radiation from massive stars (see [44], hereafter RGS08). The effect of SN explosions is somewhat model dependent and uncertain because it is treated using a subgrid recipe. Hence, the simulation analyzed in this work includes metal pollution but not mechanical feedback by SNe.

In RG05, we included the effect of reionization in the simulation 256L1p3. Because the size of the simulation box has been fixed at $\sim 1.5$ comoving Mpc, the simulation volume is too small to model the process of cosmological reionization with sufficient accuracy. We therefore assume that the simulation volume is located inside an H II region of a bright galaxy at a higher redshift. Specifically, we introduce a source of ionizing radiation within the computational box, properly biased, which corresponds to a star-forming galaxy with the constant star formation rate of 1 solar mass per year (similar to star formation rates of observed Lyman Break Galaxies at $z \sim 4$, Steidel et al. [85]). The source is switched on at $z=9.0$, and by $z=8.3$ the whole simulation box is completely ionized.

3.1.4. Summary of Main Results. The main results of the simulations are the following (see [44], for details).

(1) Negative Feedback. $\mathrm{H}_{2}$ photodissociation from FUV radiation, the main negative feedback thought to suppress the formation of the first galaxies, is not the dominant feedback. If we modify the spectrum of the sources of radiation to artificially increase or decrease the FUV flux emitted by up to one order of magnitude, we do not find any appreciable effect on the global star formation history.

(2) Self-Regulation. Feedback by hydrogen ionizing radiation (EUV) plays the key role. Galactic outflows, produced by UV photoheating from massive stars, and $\mathrm{H}_{2}$ formation/photodissociation induces a bursting star formation mode in the first galaxies that acts as the catalyst for $\mathrm{H}_{2}$ reformation inside relic (recombining) H II regions and in the "precursors" of cosmological Strömgren spheres (i.e., positive feedback regions). As a result, the formation of the first galaxies is self-regulated on a cosmological distance scale. It is significantly reduced by radiative feedback but it is not completely suppressed, even in halos with $v_{\max } \sim$ $5-10 \mathrm{~km} \mathrm{~s}^{-1}$. Note that our subgrid recipe for star formation assumes a Schmidt law with a tunable efficiency parameter $\epsilon_{*}$ (the fraction of gas converted into stars per crossing time). We find that the global star formation history and the fraction of baryons converted into stars in each galaxy, $f_{*}=M_{*} / M_{\mathrm{bar}}$, are nearly independent on the assumed value of $\epsilon_{*}$. This is typical for feedback regulated star formation. Hence, the star formation efficiency, $f_{*}$, is not an assumed parameter but it is extracted from the simulations. Thus, the derived star formation efficiency $f_{*}$ is a very generic prediction of our feedback model because it is nearly independent of the assumed value of $\epsilon_{*}$, that is instead quite uncertain. 


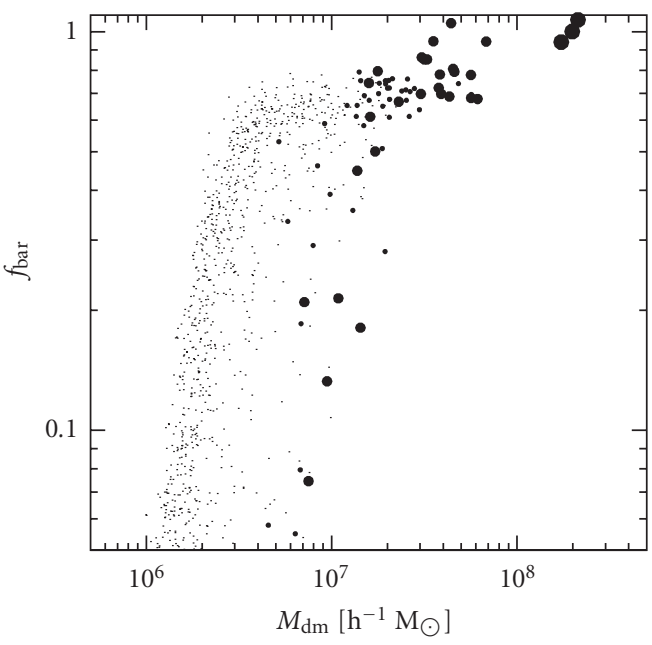

(a)

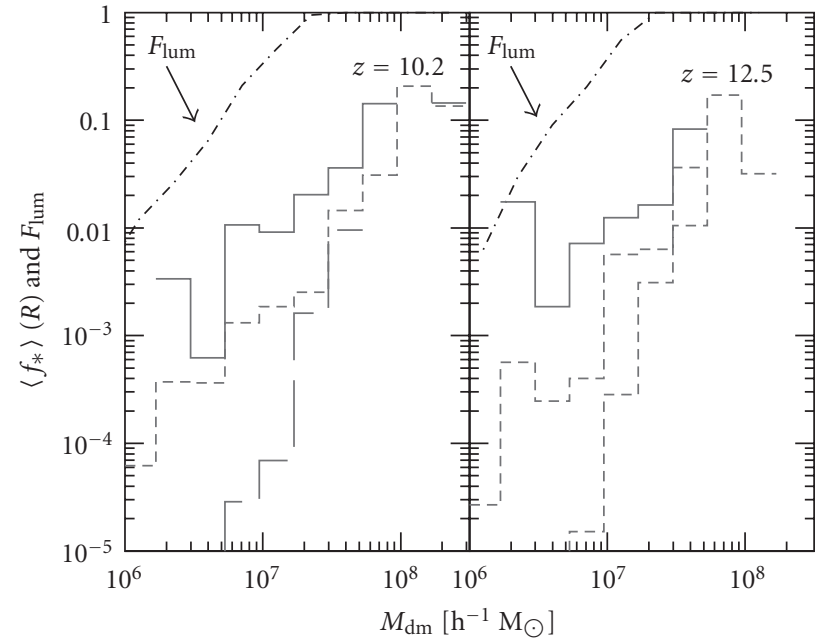

(b)

FiguRE 4: (a) Fraction of baryons retained by each galaxy (normalized to the cosmic mean value, $M_{\mathrm{bar}}=f_{\mathrm{bar}}^{\max } M_{\mathrm{dm}}$, where $f_{\mathrm{bar}}^{\max }=\Omega_{b} / \Omega_{m}=$ 0.136) as function of the halo mass $M_{\mathrm{dm}}$ for run S1 in RGS08 at $z=10$. The size of the dots is proportional to the fraction of stars $f_{*}=M_{*} / M_{\text {bar }}$ in each halo: from the largest to the smallest dots we have $f_{*}>10 \%, 1<f_{*}<10 \%, 0.1<f_{*}<1 \%$ and $f_{*}<0.1 \%$, respectively. The plot illustrates the role of internal and external sources of ionizing radiation in reducing the gas retained by small mass halos. Luminous sources (with larger $f_{*}$ ) retain less gas than dark halos due to winds driven by internal sources of radiation. (b) Average star formation efficiency $\left\langle f_{*}\right\rangle$ as a function of halo mass at $z=10.2$ (left panel) and 12.5 (right panel) for run S1. We divide all halos into three groups: those at distance $d<8 \mathrm{kpc}$ from the nearest luminous halo (solid histograms), those with $8 \mathrm{kpc}<d<50 \mathrm{kpc}$ (dashed histogram), and those with $d>50 \mathrm{kpc}$ (long-dashed histograms). The dot-dashed curve shows the fraction of luminous halos $F_{\text {lum }}\left(M_{\mathrm{dm}}\right)$ as a function of the halo mass.

(3) Contribution to Reionization. Due to the feedbackinduced bursting mode of star formation in pre-reionization dwarfs, the cosmological H II regions that they produce remain confined in size and never reach the overlap phase (e.g., Figure 1). Reionization is completed by more massive galaxies.

(4) Gas Photoevaporation and Metallicity. Star-forming dwarf galaxies show large variations in their gas content because of the combined effects of stellar feedback from internal sources and IGM reheating. Ratios of gas to dark matter lie below the cosmic mean in halos with masses $M_{\mathrm{dm}}<10^{8} \mathrm{M}_{\odot}$. Figure 4 (a) shows the fraction of baryons retained by dark and luminous halos. It is clear that small mass luminous halos lose most of their gas before reionization due to internal radiation sources. Dark halos instead are able to retain gas for a longer time (see also [2]). Gas depletion increases with decreasing redshift: the lowermass halos lose all their gas first but, as the universe evolves, larger halos with $M_{\mathrm{dm}} \sim 10^{8} \mathrm{M}_{\odot}$ also lose a large fraction of their gas. Gas photoevaporation reduces the metallicity of pre-reionization dwarfs to levels consistent with observations of dSph galaxies.

(5) Number of Luminous Galaxies. Only about 1\% of dwarf dark matter halos with mass $M_{\mathrm{dm}} \sim 5 \times 10^{6} \mathrm{M}_{\odot}$, assembled prior to reionization, are able to form stars. The fraction of luminous halos scales with the halo mass as $F_{\text {lum }} \propto M_{\mathrm{dm}}^{2}$. Thus, most halos with mass $\gtrsim 5 \times 10^{7} \mathrm{M}_{\odot}$ are luminous.
Figure $4(\mathrm{~b})$ shows $F_{\text {lum }}$ as a function of the halo mass at redshifts $z=12.5$ and $z=10.2$. The figure also illustrated that $f_{*}$ depends on the environment. Namely, it depends on the proximity of the pre-reionization dwarfs to other luminous galaxies. We find $\sim 450$ dwarf galaxies per $\mathrm{Mpc}^{3}$ with bolometric luminosity between $10^{4}$ and $10^{8} \mathrm{~L}_{\odot}$. The luminosity function is rather flat at low luminosities, with about 10 galaxies per $\mathrm{Mpc}^{3}$ in the range $10^{7}<L<10^{8} \mathrm{~L}_{\odot}$, and about $220 \mathrm{Mpc}^{-3}$ in the ranges $10^{4}<L<10^{5} \mathrm{~L}_{\odot}$ and $10^{5}<L<10^{6} \mathrm{~L}_{\odot}$.

(6) Star Formation Efficiency and Mass-to-Light. The mean star formation efficiency $\left\langle f_{*}\right\rangle=\left\langle M_{*} / M_{\mathrm{bar}}^{\max }\right\rangle$, where $M_{\mathrm{bar}}^{\max } \simeq$ $M_{\mathrm{dm}} / 7$, is found to be nearly independent of redshift and to depend on total mass as $\left\langle f_{*}\right\rangle \propto M_{\mathrm{dm}}^{\alpha}$ with $\alpha=2$ if the radiative feedback is strong (i.e., top heavy IMF and/or large $\left\langle f_{\text {esc }}\right\rangle$ ) and $\alpha=1.5$ if the feedback is weak. This is shown in Figure 5(a), where we plot the mean star formation efficiency, $\left\langle f_{*}\right\rangle$ and the mean gas fraction $\left\langle f_{g}\right\rangle$ in halos of mass $M_{\mathrm{dm}}$.

(7) Scatter of the Mass-to-Light Ratio. A tight relationship between the star formation efficiency $f_{*}$ and the total mass of halos holds only for galaxies with $M_{\mathrm{dm}}>5 \times 10^{7} \mathrm{M}_{\odot}$. In lower-mass halos, the scatter around the mean $\left\langle f_{*}\right\rangle$ is increasingly large (see Figure 5(b)). For a given halo mass, the galaxy can be without stars (dark galaxy) or have star formation efficiency $f_{*} \sim 0.1$. However, only a few dark galaxies with mass at formation $M_{\mathrm{dm}} \sim 1-5 \times 10^{7} \mathrm{M}_{\odot}$ should exist in the Local Group. 


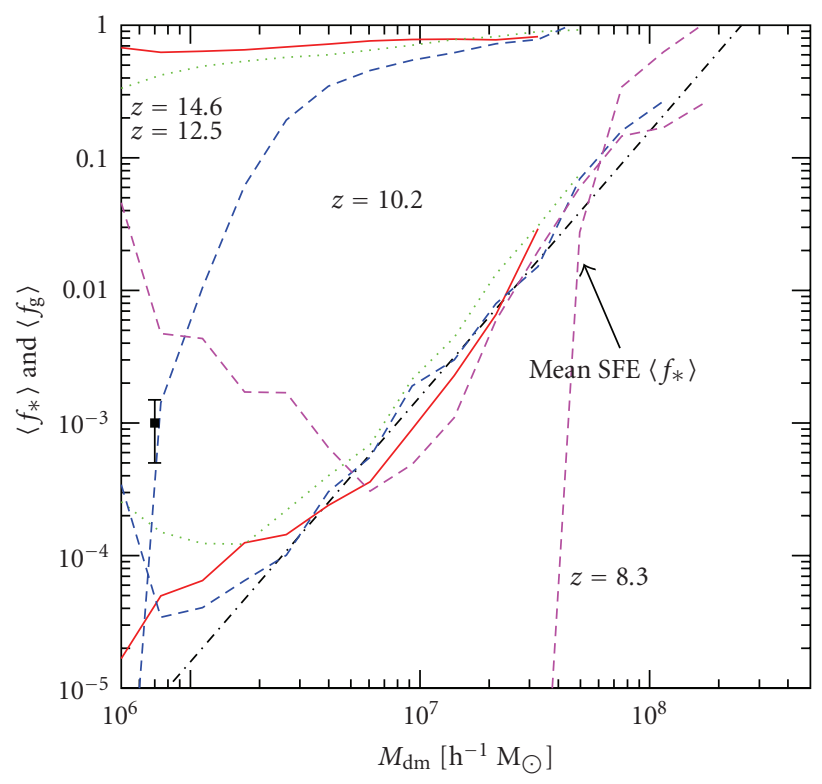

(a)

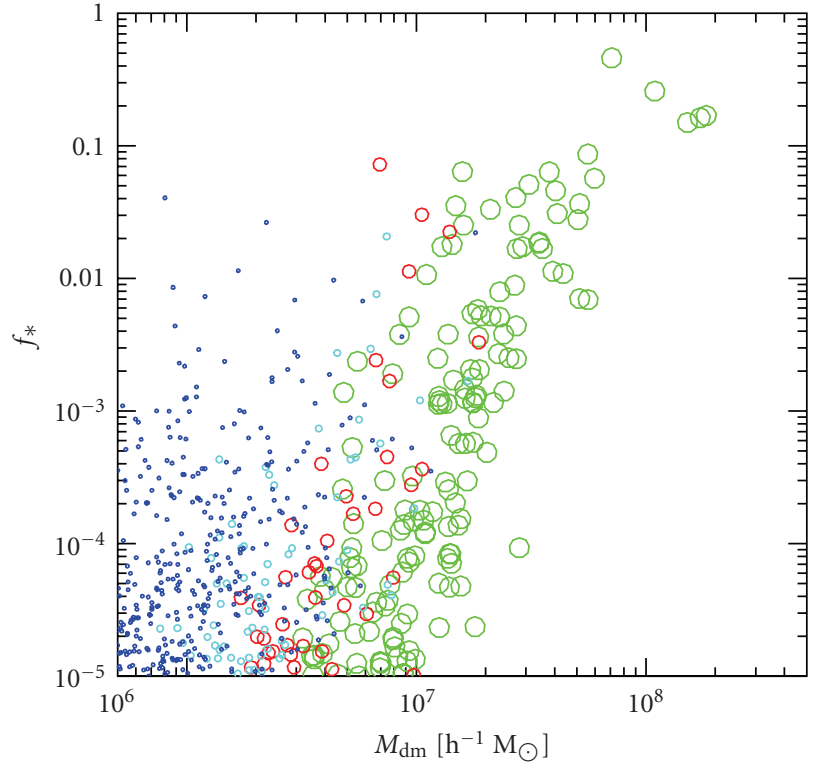

(b)

Figure 5: (a) Average star formation efficiency (i.e., fraction of the collapsed baryon mass converted into stars), $\left\langle f_{*}\right\rangle=\left\langle M_{*} / M_{\mathrm{bar}}\right\rangle($ thick curves), and gas fraction (i.e., fraction of the collapsed baryon mass retained in the gas phase) $\left\langle f_{\mathrm{g}}\right\rangle=\left\langle M_{\mathrm{g}} / M_{\mathrm{bar}}\right\rangle$ (thin curves), of the first galaxies as a function of their halo mass for run S2 in RGS08 at $z=14.5,12.5,10.2$, and 8.3. For comparison, the symbol with error bar shows the expected star formation efficiency (roughly $M_{*} \sim 30-300 \mathrm{M}_{\odot}$ divided by the baryonic mass of the halo) in the first mini halo of mass $10^{6} \mathrm{M}_{\odot}$ simulated by Abel et al. [71]. The dot-dashed line shows a power-law fit for the mean SFE, $\left\langle f_{*}\right\rangle\left(M_{\mathrm{dm}}, z\right) \propto M_{\mathrm{dm}}^{2}$. The SFE is nearly independent of redshift apart from an increase in halos with $M_{\mathrm{dm}}<10^{7} \mathrm{M}_{\odot}$ at $z \sim 8$. (b) Same as (a) but showing the star formation efficiency, $f_{*}$, for individual galaxies in the simulation at $z=10.2$. Circles, from smaller to the larger, refer to galaxies with gas fractions $f_{\mathrm{g}}<0.1 \%$ (blue), $0.1 \%<f_{\mathrm{g}}<1 \%$ (cyan), $1 \%<f_{\mathrm{g}}<10 \%$ (red), and $f_{\mathrm{g}}>10 \%$ (green), respectively.

(8) Size and Morphology of Stellar Component. Galaxies with masses $M_{\mathrm{dm}}<10^{8} \mathrm{M}_{\odot}$ have a low surface brightness and extended stellar spheroid. At $z \sim 10$, the outer edges of the stellar spheroid nearly reach the virial radius. In more massive galaxies that cool more efficiently by Lyman-alpha radiation, the stars and light are more centrally concentrated. Figure 6 shows the structural properties of the dark matter and stellar halo in three of the most massive galaxies in our simulation. These dwarf galaxies have properties similar to Draco and Umi dSphs. The figure also shows that the velocity dispersion of the stars in these dwarfs is about a factor of two smaller than $v_{\max }$.

3.1.5. Photoevaporation and Reionization Feedback. The small total mass of the first galaxies has two other implications. First, the ionizing radiation emitted by massive stars can blow out most of the gas before SN-driven winds become important, further reducing star formation rates (see RGS08). Second, the increase in temperature of the IGM to $10,000-20,000 \mathrm{~K}$ due to $\mathrm{H} \mathrm{I}$ reionization prevents the gas from condensing into newly virialized halos with circular velocities smaller than $10-20 \mathrm{~km} \mathrm{~s}^{-1}$ (e.g., [2, 27, 28, 35, 40, 86]). It follows that dwarf galaxies with $v_{\max }<10-20 \mathrm{~km} \mathrm{~s}^{-1}$ lose most of their gas before reionization and stop accreting new gas and forming stars after reionization.

The value $v_{\max } \sim 20 \mathrm{~km} \mathrm{~s}^{-1}$ that we use to define a fossil is motivated by the fundamental differences in cooling and feedback processes discussed above that regulate star formation in the early universe. It is not the critical value for suppression of gas accretion due to reionization. Indeed, we discuss in Section 4 that pre-reionization fossils may have a late phase of gas accretion and star formation well after reionization, at redshift $z<1-2$. Thus, a complete suppression of star formation after reionization (about 12 Gyr ago) is not the defining property of a fossil dwarf.

\section{Late Time Cold Accretion from the IGM}

The ability of the IGM gas to condense at the center of dark halos depends on the ratio, $\Gamma=v_{\text {vir }} / c_{s, \text { igm }}$, of the circular velocity to the IGM sound speed and also on the dark halo concentration, $c$ [3]. Typically, the concentration of a halo is $\mathcal{c}_{\text {vir }} \sim 4$ at the redshift of virialization $[87,88]$ but, as the halo evolves in the expanding universe, its concentration increases $\propto\left(1+z_{\text {vir }}\right) /(1+z)$. The evolution of the halo concentration with redshift can be understood in the context of the theory of cosmological secondary infall of dark matter [89] and has been quantified using N-body simulations [87, 88]. Thus, primordial halos with $v_{\text {vir }}<10-20 \mathrm{~km} \mathrm{~s}^{-1}$ stop accreting gas immediately after reionization, but, in virtue of their increasing concentration and the decreasing temperature of the IGM at $z<3$ (after He II reionization), they may start accreting gas from the IGM at later times (see [3]). As a result, we expect that if the fossils of the first galaxies exist in 


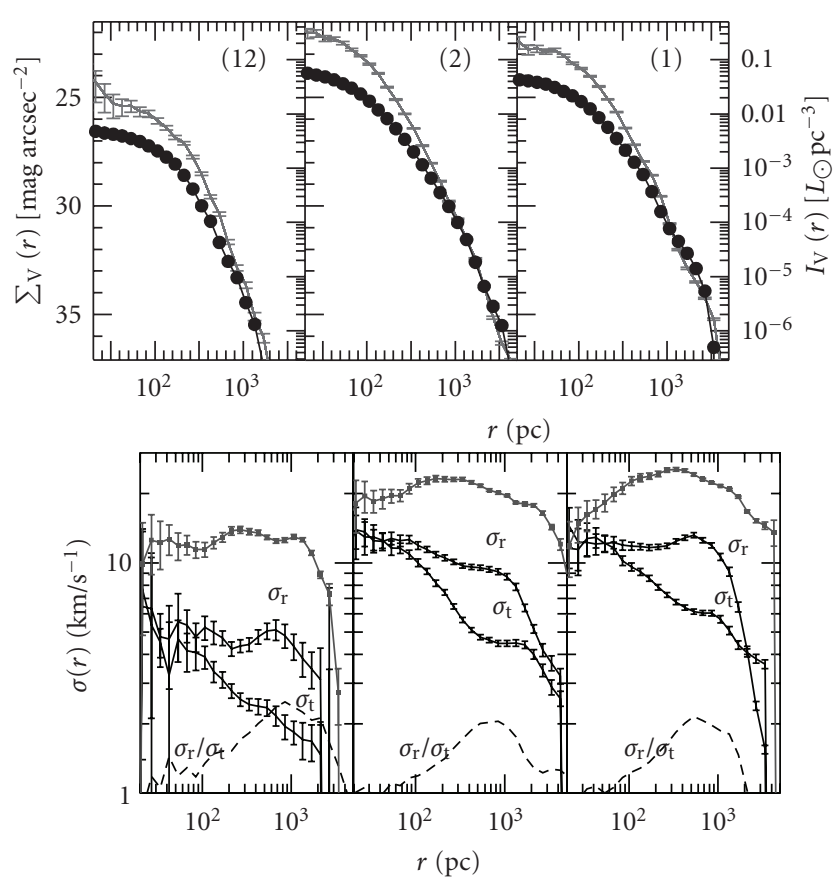

Figure 6: The top panels show the surface brightness (black point) and luminosity density (gray points) radial profiles for 3 galaxies from among the most massive dwarf galaxies extracted from the RG05 simulation at $z=8.3$. We have evolved the stellar population passively to $z=0$. The simulated galaxies shown in this figure have a stellar spheroid similar to Draco and Umi already in place at the time of formation (i.e., the spheroid is not produced by tidal effects). The bottom panels show the velocity dispersion profiles of the dark matter (gray points) and of the stars (black points) for the same 3 galaxies. The velocity dispersion of the stars is split in the radial and tangential components. All quantities are spherically averaged because the dark matter and stars have nearly spherical symmetry.

the Local Group (RG05), they may have a more complex star formation history than previously envisioned. A signature of this model is a bimodal star formation history with an old ( $\sim 13 \mathrm{Gyr})$ and a younger $(\lesssim 5-10 \mathrm{Gyr}$, depending on the halo mass) population of stars. Leo $\mathrm{T}$ properties can be reproduced by this simple model for late gas accretion [3]. In addition, Leo $\mathrm{T}$ seems to show a bimodal star formation history [68] as expected in our model. Still, other models may also explain the observed star formation history of Leo $\mathrm{T}[90]$.

Perhaps more important is the possible existence of dark galaxies: small mass halos containing only gas but no stars. Dark galaxies are most likely to exist if pre-reionization fossils do not form efficiently due to dominant negative feedback in the early universe (e.g., $\mathrm{H}_{2}$ photodissociation by the FUV background).

The late gas accretion from the IGM is shown in Figure 7 for dark halos with circular velocity at virialization $v_{\text {vir }}=$ $18,15,12,9$ and $6 \mathrm{~km} \mathrm{~s}^{-1}$ (lines from the top to the bottom). The lines show the evolution of the gas density in the core of the halo as a function of redshift. The core radius is typically $100 \mathrm{pc}$ and the labels show the circular velocity at

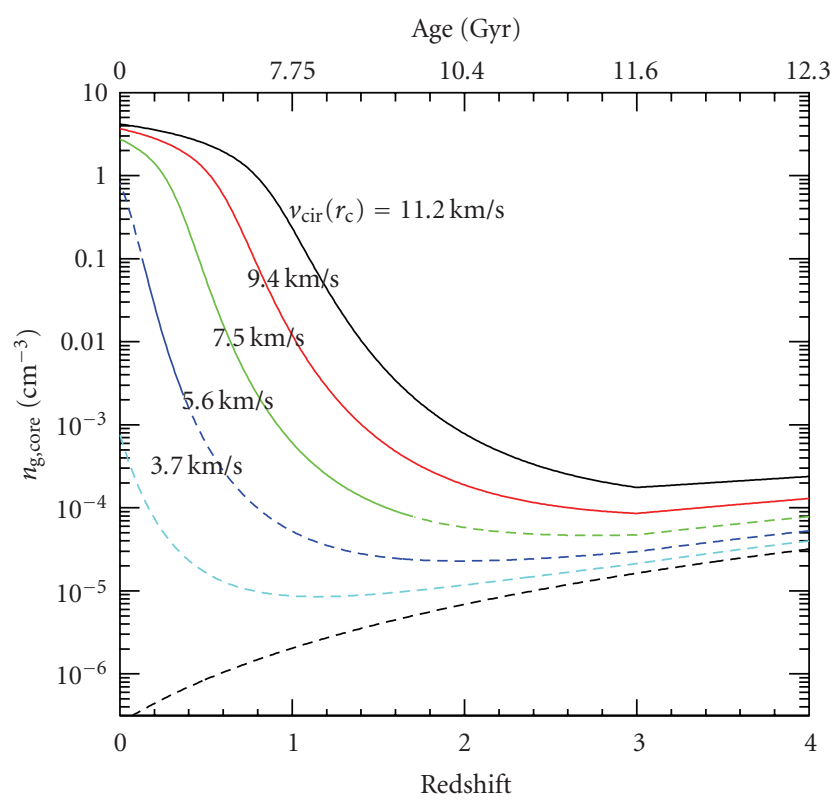

FIGURE 7: The evolution of the gas density in the core of dark halos due to cold accretion from the IGM for halos with $v_{\text {vir }}=18,15,12,9$ and $6 \mathrm{~km} \mathrm{~s}^{-1}$ (from the top to the bottom), and corresponding to the circular velocities at the core radius $v_{\text {cir }}\left(r_{\text {core }}\right)=0.66 v_{\text {vir }}$ (shown by the labels). The curves are assuming isothermal equation of state of the gas but the dashed portions show the parameter space in which such assumption fails because the gas cannot cool sufficiently fast as it is compressed toward the center of the halo.

the core radius $\left(v_{\text {cir }}\left(r_{\text {core }}\right) \approx 0.66 v_{\text {vir }} \approx 0.624 v_{\text {max }}\right.$, where $v_{\text {vir }}$ is circular velocities at the virial radius and $v_{\max }$ is the maximum circular velocity). We show the evolution of the gas density only for halos that are affected by reionization feedback. More massive halos will also accrete gas from the IGM as they evolve in isolation after virialization, but the gas accretion is continuous and not affected by reionization.

Under the scenario in which halos with masses smaller than the critical value of $10^{8}-10^{9} \mathrm{M}_{\odot}$ remain dark due to feedback effects, the increase in their dark matter concentration and the temperature evolution of the IGM will produce a late phase of gas accretion at redshift $z<1-2$. If the gas has very low metallicity or is metal free, it is unlikely that the accreted gas will be able to form stars in the smallest mass halos. This is why we envisioned the possible existence of dark galaxies. However, their mass would be smaller than the mass of any luminous galaxy and their discovery would be challenging.

The level of metal pre-enrichment necessary to initiate star formation in minihalos that experience a late phase of gas accretion can be roughly estimated from the cooling function from hyperfine transitions of oxygen and carbon: $\Lambda_{23} \sim 10^{-3}\left(Z / Z_{\odot}\right)$, where $\Lambda_{23}=10^{-23} \mathrm{erg} \mathrm{s}^{-1} \mathrm{~cm}^{3}$ and $Z$ is the gas metallicity. A necessary condition for star formation is $t_{\text {cool }} \approx(0.7 \mathrm{yr}) T /\left(n_{\mathrm{g}, \text { core }} \Lambda_{-23}\right)<t_{\mathrm{H}}$, that can be written as $n_{\mathrm{g}, \text { core }}>0.03 \mathrm{~cm}^{-3}\left(Z / 10^{-2} \mathrm{Z}_{\odot}\right)^{-1}$. Figure $8(\mathrm{a})$ shows $n_{\mathrm{g}, \text { core }}$ and $N_{\mathrm{H}}$ in minihalos that evolve isothermally at $T \sim 10^{4} \mathrm{~K}$ but that do not form stars (i.e., candidates for extragalactic 


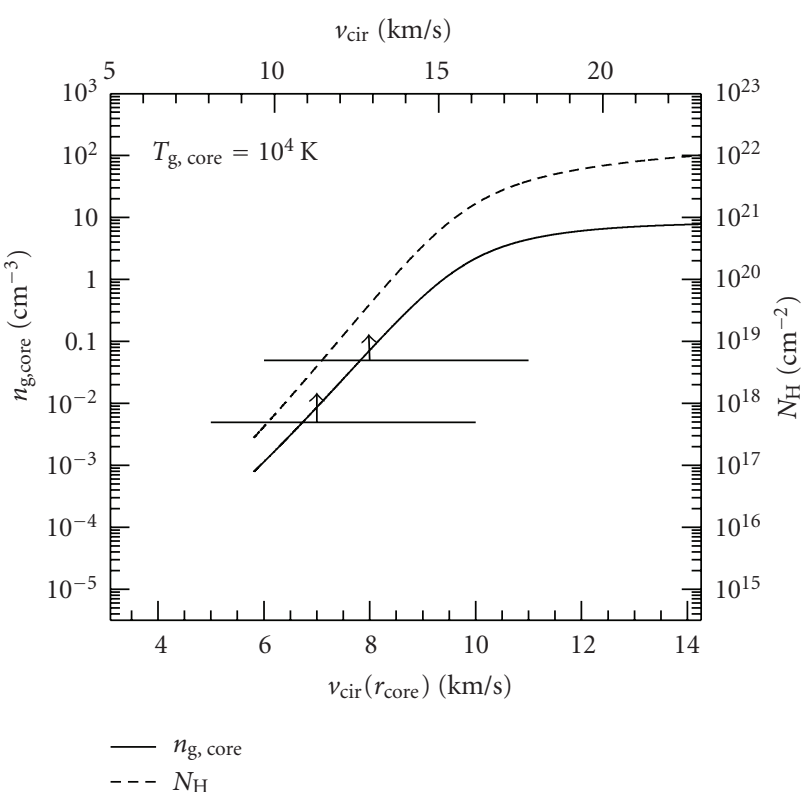

(a)

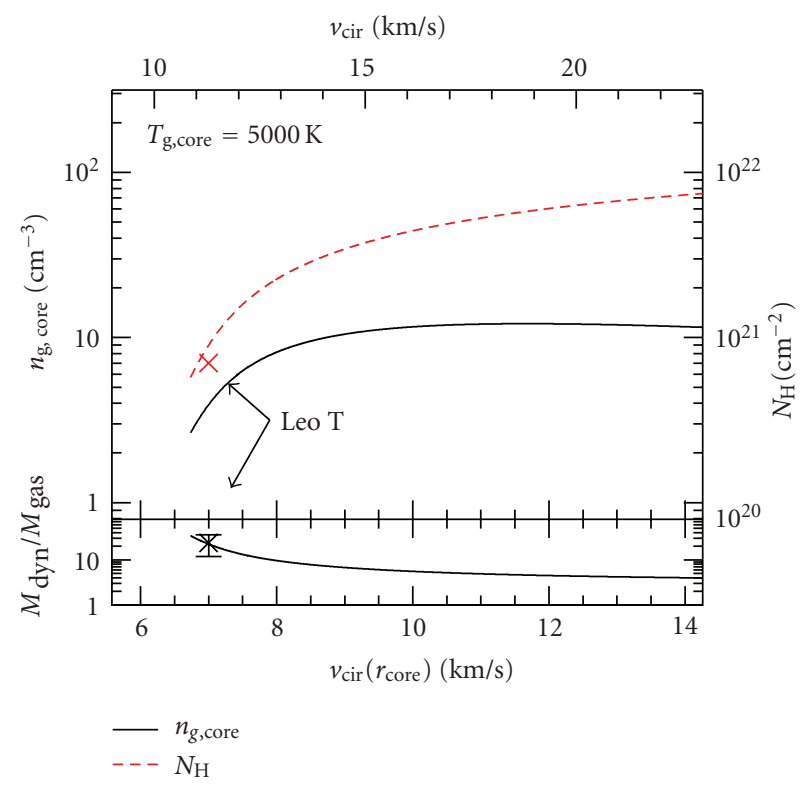

(b)

Figure 8: (a) The gas density, $n_{\mathrm{g}, \text { core }}$ (solid curves), and hydrogen column density, $N_{\mathrm{H}}=2 r_{\mathrm{c}} n_{\mathrm{g}, \text { core }}$ (dashed curves), within the core, $r_{\mathrm{c}}$, of a minihalo at redshift $z=0$ as a function its circular velocity at $r_{\mathrm{c}}$. The minimum $v_{\text {cir }}$ in each curve is determined by the condition $t_{\text {rec }} / t_{\mathrm{H}}<1$, necessary for cooling to $T_{\text {gas }} \sim 10^{4} \mathrm{~K}$. The horizontal lines with arrows show the requirement for cooling to temperatures below $10^{4} \mathrm{~K}$, necessary for initiate star formation, for gas metallicity $Z=0.1$ (lower line) and $0.01 \mathrm{Z}_{\odot}$ (higher line). (b) Same as (a) but for minihalos whose gas is able to cool to $T_{\text {g,core }}=5000 \mathrm{~K}$ due to metal pre-enrichment $\left(Z=0.1 \mathrm{Z}_{\odot}\right)$. These halos are likely able to sustain a multi-phase ISM and form stars.

CHVCs and dark galaxies). The horizontal lines show the requirement for metal cooling and star formation assuming gas metallicity $Z=0.1$ and $0.01 \mathrm{Z}_{\odot}$. Figure $8(\mathrm{~b})$ shows $n_{\mathrm{g} \text {,core, }}$, $N_{\mathrm{H}}$ and $M_{\text {dyn }} / M_{\text {gas }}$ (the dynamical mass to gas mass ratio) in the core of minihalos that are able to cool to $T_{\mathrm{g}, \text { core }}=5000 \mathrm{~K}$ (roughly the temperature of the ISM in Leo T), and thus form stars. The symbols show the observed value for Leo $\mathrm{T}$.

\section{Comparison of Theory and Observations}

5.1. Number of Fossils and Nonfossil Satellites in the Milky Way. N-body simulations can be used to estimate the number of dark halos in the Milky Way with maximum circular velocity $v_{\max }>20 \mathrm{~km} \mathrm{~s}^{-1}$. However, there is a complication to this naive calculation. A significant fraction of dark halos that today have $v_{\max }<20 \mathrm{~km} \mathrm{~s}^{-1}$ were once more massive, due to tidal stripping [91]. According to our definition, dwarf galaxies formed in these dark halos would not be pre-reionization fossils if they had at any time during their evolution $v_{\max }(t)>20 \mathrm{~km} \mathrm{~s}^{-1}$ (see Section 3.1.5). If the number of observed Milky Way satellites exceeds the estimated number of these massive halos we must conclude that at least a fraction of the observed Milky Way satellites are pre-reionization fossils.

However, there is an assumption in this scenario. One must assume that the stars in these halos survive tidal stripping for as long as the dark matter. In this case tidally stripped halos may indeed account for a fraction or all of the newly discovered ultrafaint dwarfs. However, Peñarrubia et al. [60] find that tidally stripped dark halos lose their stars more rapidly than they lose their dark matter. Thus, they may become dark halos even though they were initially luminous satellites. These dark halos should not be counted as ultrafaint dwarfs.

Using results of published N-body simulations of the Milky Way, Bovill and Ricotti [51] have estimated the number of dark halos that have or had in the past $v_{\max }(t)>$ $20 \mathrm{~km} \mathrm{~s}^{-1}$ (i.e., non pre-reionization fossils). In Table 1 we summarize the results of the counts for dark matter and luminous satellites for two large N-body simulations of a Milky Way type halo: the "Aquarius" simulation [92] and the Via Lactea I simulation [93].

The number of luminous satellites that exist within the Milky Way is highly uncertain beyond a distance from the Galactic center of $200 \mathrm{kpc}$. Tollerud et al. [25], after applying incompleteness corrections, estimated 304-576 satellites within $417 \mathrm{kpc}$ and about 176-330 within $200 \mathrm{kpc}$ (the numbers are from their Table 3). As shown in Table 1, the existence of some pre-reionization fossils among the ultrafaint dwarfs appears to be favored by the data. However, the current uncertainties on the completeness corrections of observations and on the simulations are too large to deem the existence of fossils as necessary.

The error bars on the theoretical estimate of the number of fossils in the Milky Way shown in Table 1 come from uncertainties in the fraction of halos that were more massive in the past. This fraction was derived from simulations by [91]. Another uncertainty in the simulation results can 
TABLE 1: Number of observed satellites versus number of dark halos with $v_{\max }(t)>20 \mathrm{~km} \mathrm{~s}^{-1}$ (i.e., non pre-reionization fossils) for the Milky Way.

\begin{tabular}{|c|c|c|c|c|c|}
\hline \multirow{3}{*}{ Distance from center } & \multirow{3}{*}{ Luminous dwarfs } & \multicolumn{4}{|c|}{ Dark halos with $v_{\max }(t)>20 \mathrm{~km} \mathrm{~s}^{-1}$ via } \\
\hline & & \multicolumn{2}{|c|}{ Lactea I sim. } & \multicolumn{2}{|c|}{ Aquarius sim. } \\
\hline & & Today & Any time & Today & Any time \\
\hline$<200 \mathrm{kpc}$ & 176 to 330 & 14 & $36 \pm 8$ & 34 & $91 \pm 20$ \\
\hline$<417 \mathrm{kpc}$ & 304 to 576 & 28 & $73 \pm 16$ & 69 & $182 \pm 40$ \\
\hline
\end{tabular}

be attributed to the different predictions for the number of Milky Way satellites in the Via Lactea I and II and Aquarius simulations. The discrepancy can be partially attributed to different cosmology in the simulations but mostly because the Via Lactea I simulation likely used erroneous initial conditions. Finally, Tollerud et al. [25] corrections on the number of observed satellites also rely on the radial distribution of dark matter sub halos extracted from Via Lactea I simulations that may be erroneous. Once the discrepancies among different simulations are better understood the number of simulated satellites of the Milky Way may be known with greater certainty.

Using comparisons between the predicted and observed Galactocentric distributions of dwarf satellites around the Milky Way, Gnedin and Kravtsov [94], hereafter GK06, have estimated that pre-reionization fossils may constitute about $1 / 3$ of Milky Way dwarfs. GK06 estimated the number of fossils in the Milky Way using data from the simulations of the first galaxies in RG05. GK06 defined a fossil as a simulated halo which survives at $z=0$ and remains below the critical circular velocity of $20 \mathrm{~km} \mathrm{~s}^{-1}$ with no appreciable tidal stripping (the usual definition of fossil adopted in this paper as well). They calculate the probability, $P_{S}\left(v_{\max }, r\right)$, of a luminous halo with a given maximum circular velocity $v_{\max }$ to survive from $z=8$ (the final redshift of the RG05 simulation) to $z=0$. The surviving halos are assigned a luminosity based on the $L_{\mathrm{V}}$ versus $v_{\max }$ relationship from RG05. At $z=0$, GK06 has a population of dwarf galaxies with a resolution limit of $v_{\max }=13 \mathrm{~km} \mathrm{~s}^{-1}$. This limit corresponds to a lower luminosity limit of $L_{\mathrm{V}} \sim 10^{5} \mathrm{~L}_{\odot}$, which includes Leo $\mathrm{T}$ and Canes Venatici I, but excludes all the other new ultrafaint Milky Way satellites.

In Figure 9, we show the cumulative luminosity function from GK06 for the Milky Way and M31 satellites. Figure 9(c) shows satellites with distance from their host $d<100 \mathrm{kpc}$, Figure 9(b) $d<300 \mathrm{kpc}$, and Figure 9(a) $d<1 \mathrm{Mpc}$. The gray lines show the GK06 predictions, and the shaded region encompasses the error bars. The resolution limits in GK06 cause halos with $v_{\max }<17 \mathrm{~km} \mathrm{~s}^{-1}$ to be preferentially destroyed by tidal effects. The dashed line show the predicted luminosity function corrected for the resolution effects. Both the uncorrected (solid lines) and corrected (dashed lines) luminosity functions are plotted in Figure 9(c). The points with error bars show the observed luminosity function of $\mathrm{dSph}$ galaxies around the Milky Way and M31 corrected only for limits in sky coverage of the SDSS survey. The plot is from GK06 but has been updated to include the new ultrafaint dwarfs with $L_{\mathrm{V}} \gtrsim 10^{5} \mathrm{~L}_{\odot}$.
The results of this model are consistent with the observations. The model reproduces the Galactocentric distribution of the most luminous dSphs, even though in this model dSphs are not tidally stripped dIrrs. It also shows a good agreement with observations for luminosities that can be considered nearly complete within a given Galactocentric distance.

5.2. Statistical Properties of Pre-Reionization Fossils. In this section, we compare the properties of the new dwarf galaxies discovered in the Local Group to the theoretical predictions of simulations of primordial galaxies formed before reionization. The argument that justifies this comparison is that star formation stops or is greatly reduced after reionization (but see Section 4). We do not expect two perfectly distinct populations of fossil galaxies with $v_{\max }<20 \mathrm{~km} \mathrm{~s}^{-1}$ and nonfossils with $v_{\max } \geq 20 \mathrm{~km} \mathrm{~s}^{-1}$, but a gradual transition of properties from one population to the other. Some fossils may become more massive than $v_{\max } \sim 20 \mathrm{~km} \mathrm{~s}^{-1}$ after reionization, accrete some gas from the IGM, and form a younger stellar population. These dwarfs are no longer defined as "fossils". However, if the dark halo circular velocity remains close to $20 \mathrm{~km} \mathrm{~s}^{-1}$ the young stellar population is likely to be small with respect to the old one. In RG05 we call these galaxies "polluted fossils" because they have the same basic properties of "fossils" with a sub-dominant young stellar population. A similar argument can be made regarding the late phase of gas accretion that may produce objects similar to Leo T.

In Figures 10 and 11, we compare the RG05 predictions for the fossils of primordial galaxies to the observed properties of the new Milky Way and M31 dwarfs. The symbols and lines in Figures 10 and 11 have the following meanings. All known Milky Way dSphs are shown by circles; Andromeda's dSphs satellites are shown by triangles; simulated fossils are shown by the small solid squares. The solid and open symbols refer to previously known and new dSphs, respectively. The transition between fossils and nonfossil galaxies is gradual. In order to illustrate the different statistical trends of "nonfossil" galaxies we show dwarf irregulars (dIrr) as asterisks and the dwarf ellipticals (dEs) as crosses, and we show the statistical trends for more luminous galaxies as thick dashed lines on the right side of each panel.

Figure 10(a) shows how the surface brightness (top panel) and half light radius (bottom panel) of all known Milky Way and Andromeda satellites as a function of V-band luminosity compares to the simulated fossils. The surface 


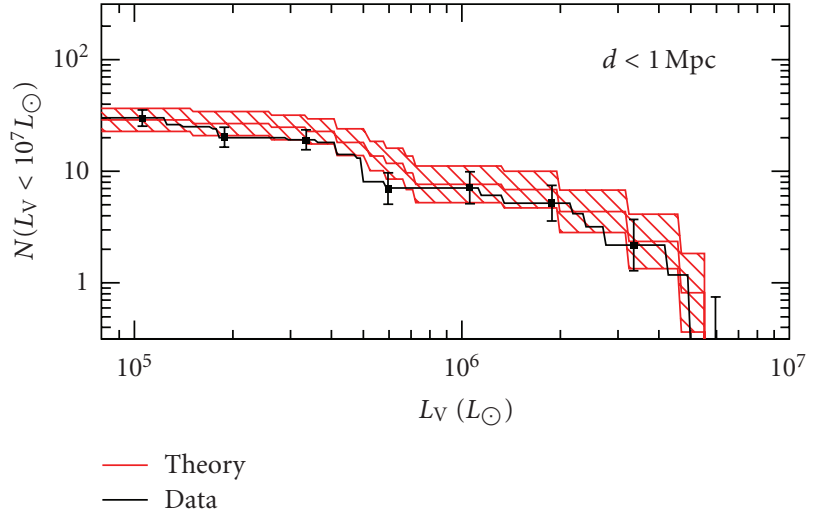

(a)

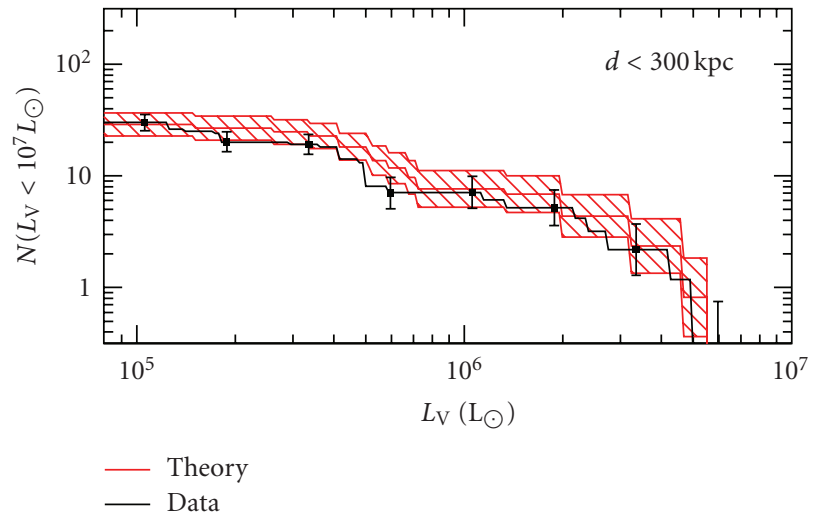

(b)

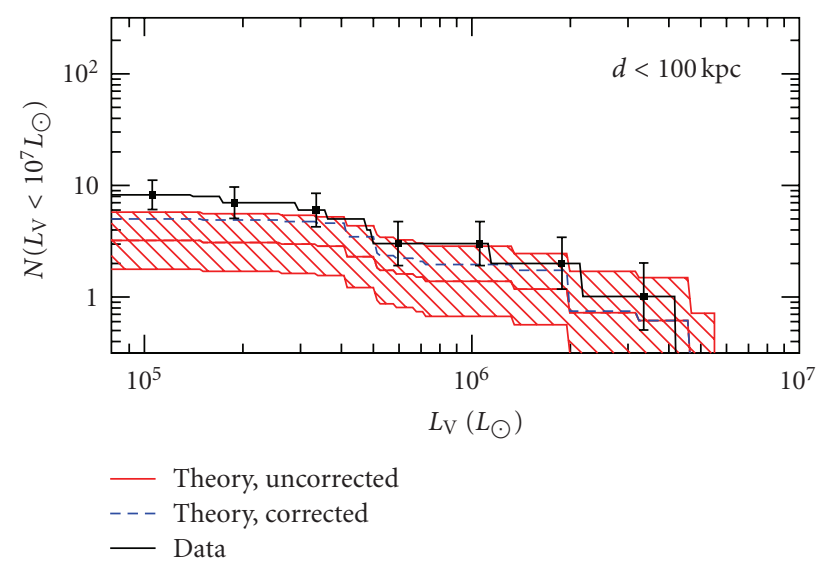

(c)

FIgURE 9: Luminosity function of pre-reionization fossil dwarfs predicted in GK06 (red bands) plotted with the luminosity function for Local Group dSphs (points with error bars). The data from observations are corrected only for limits in sky coverage of the SDSS survey.

brightness limit of the SDSS is shown by the thin solid lines in both panels of the figure. The new dwarfs agree with the predictions up to this threshold, suggesting the possible existence of an undetected population of dwarfs with $\Sigma_{\mathrm{V}}$ below the SDSS sensitivity limit. The new M31 satellites have properties similar to their previously known Milky Way counterparts (e.g., Ursa Minor and Draco). Given the similar host masses and environments, further assuming similar formation histories for the halos of M31 and the Milky Way, we may be tempted to speculate on the existence of an undiscovered population of dwarfs orbiting M31 equivalent to the new SDSS dwarfs.

The large mass outflows due to photo-heating by massive stars and the subsequent suppression of star formation after an initial burst make reionization fossils among the most dark matter dominated objects in the universe, with predicted mass-to-light ratios as high as $10^{4}$ and $L_{\mathrm{V}} \sim$ $10^{3}-10^{4} \mathrm{~L}_{\odot}$. Figure 10 (b) shows the velocity dispersion (bottom panel) and mass-to-light ratios, $M_{\sigma} / L_{\mathrm{V}}$ (top panel), as a function of V-band luminosity of the new and old dwarfs from observations in comparison to simulated fossils. The symbols are the same as in the previous figures. Theoretical and observed dynamical masses are calculated from the velocity dispersions of stars (i.e., $M_{\sigma}=2 r_{1 / 2} \sigma^{2} / G$ ), and do not necessarily reflect the total mass of the dark halo at virialization.

Observations show that the value of the dynamical mass within the stellar spheroid, $M \sim(1 \pm 5) \times 10^{7} \mathrm{M}_{\odot}$, remains relatively constant as a function of $L_{\mathrm{V}}$ [58]. Recent work by Strigari et al. [97] shows analogous results to the one found by Mateo [58]. The dynamical mass of dwarf spheroidals within a radius of $300 \mathrm{pc}$ is relatively constant: $M \sim 10^{7} \mathrm{M}_{\odot}$. The radii of the stellar spheroids in these dwarf galaxies may be either larger or smaller than $300 \mathrm{pc}$. In the later case, the determination of the mass of the dwarfs is uncertain.

Our simulation provides some insight into the reason why the dynamical mass remains roughly constant in dSphs. The simulations show that in pre-reionization dwarfs, the ratio of the radius of the stellar spheroid to the virial radius of the dark halo decreases with increasing dark halo mass (i.e., the stellar profile becomes more concentrated for more luminous dwarfs). Thus, as the halo mass and virial radius increases, the stellar spheroid becomes increasingly concentrated in the deepest part of the potential well. If follows that the ratio, $f_{\sigma} \equiv M_{\sigma} / M_{\mathrm{dm}}$, of the dynamical mass within the largest stellar orbits to total dark matter mass is 

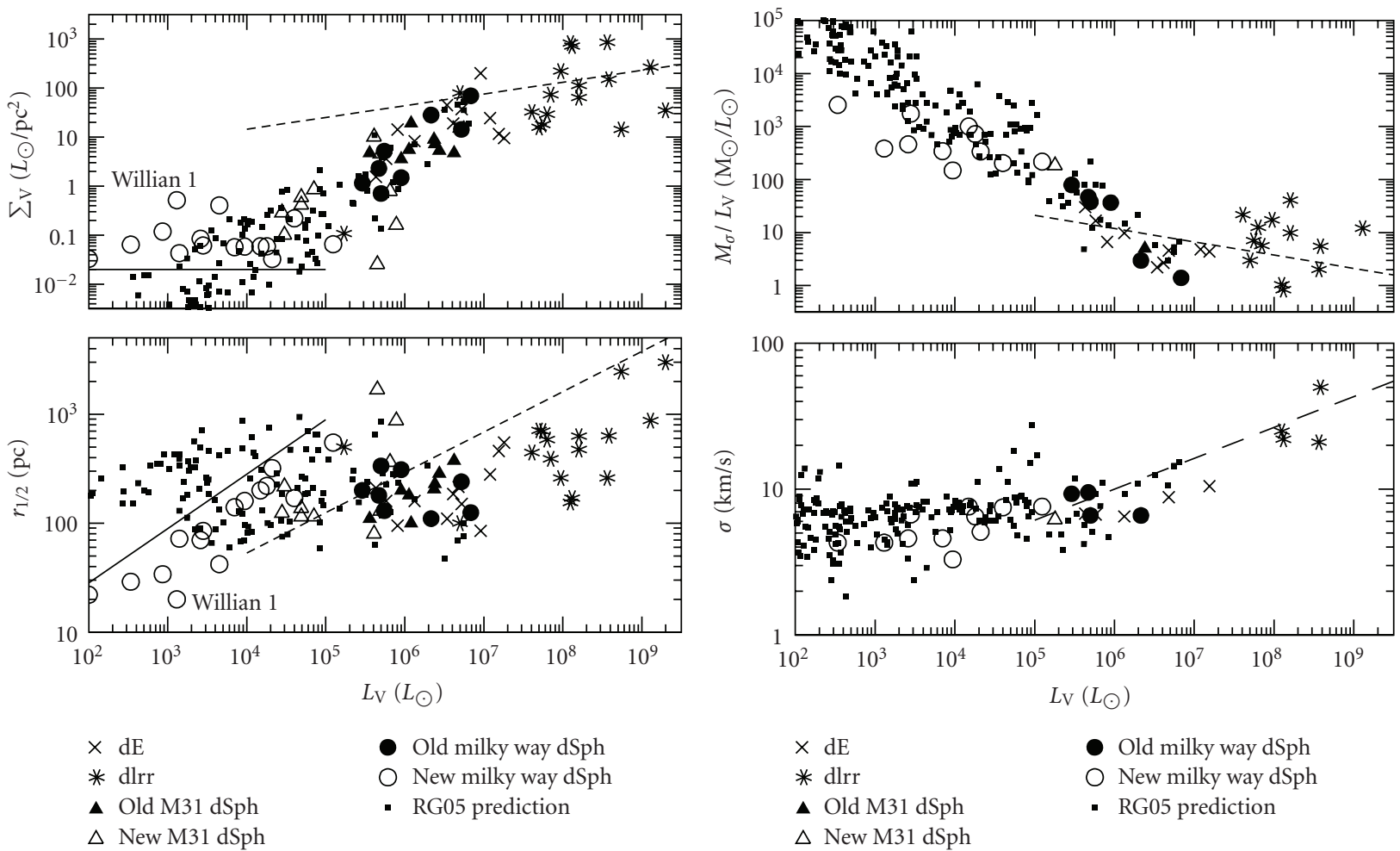

(a)

(b)

Figure 10: (a) Surface brightness and core radius versus. V-band luminosities. Small filled squares are simulated pre-reionization fossils from RG05, asterisks are dIrrs, crosses are dEs, closed circles are the previously known dSph around the Milky Way, closed triangles are previously known dSph around M31, and open circles and triangles are new dSph around the Milky Way and M31 respectively. The solid lines roughly show the detection limits inherent to the methods used to find the ultrafaints in the SDSS data [24] and the dashed lines show the scaling relationships for more luminous Sc-Im galaxies $\left(10^{8} \mathrm{~L}_{\odot} \lesssim L_{\mathrm{B}} \lesssim 10^{11} \mathrm{~L}_{\odot}\right)$ derived by Kormendy and Freeman [95]. (b) Mass-to-light ratio and velocity dispersion of a subset of the new dwarfs $[23,96]$ versus V-band luminosity. The symbols and lines are as in (a).

also reduced. Thus, the decrease of $f_{\sigma}$ for increasing dark matter mass of halos maintains the value of the dynamical mass $M_{\sigma}=f_{\sigma} M_{\mathrm{dm}}$ (measured by the velocity dispersion of the stars) almost constant, even though the total mass of the halo increases. The extent of the stellar spheroids in the lowest mass dwarfs is comparable in size to their virial radii at formation (see Section 3.1).

The metallicity-luminosity relation of the observed and simulated dwarfs is shown in Figure $11(\mathrm{a}) .[\mathrm{Fe} / \mathrm{H}]$ is plotted against V-band luminosity in solar units. Symbols for the previously known dwarfs, the new, ultrafaint dwarfs, and simulated fossils are the same as in Figure 10. In this plot we color code simulated fossils according to their star formation efficiency, $f_{*}$. Red symbols show simulated dwarfs with $f_{*}<$ 0.003 , blue $0.003 \leq f_{*} \leq 0.03$ and green $f_{*}>0.03$.

Using the data for the metallicity collected in Bovill and Ricotti [51], the new ultrafaint dwarfs do not appear to follow the tight luminosity-metallicity relationship observed in more luminous galaxies (although error bars are large). Note that here, as well as in Bovill and Ricotti [51] (although Table 3 in that paper was erroneously not updated), we have plotted data from Kirby et al. [98] for the 6 ultrafaint Milky Way satellites provided in that paper. There are several physical mechanisms that may produce the observed scatter in metallicities of dwarfs at a given constant luminosity. The large spread of star formation efficiencies producing a dwarf of a given luminosity in our simulations is responsible for at least part of the large spread of the luminositymetallicity relation. At this point it is unclear whether our simulations can reproduce the scatter of metallicities of simulated fossils, or if perhaps the luminosity of the lowest luminosity ultrafaint dwarfs has been reduced due to tidal interactions. As mentioned before we have suggested that the lowest luminosity ultrafaint dwarfs have not yet been discovered because their surface brightness lies below the SDSS detection limits.

Figure 11(b) shows the scatter of the metallicity of the stars, $\sigma_{[\mathrm{Fe} / \mathrm{H}]}$, plotted against V-band luminosity and $[\mathrm{Fe} / \mathrm{H}]$ respectively. The various point types and colors are the same used in Figure 11(a). The large spread in the metallicity of the stars is a natural consequence of the hierarchical assembly of dwarf galaxies in cosmological simulations. It is not necessarily an indication that star formation in dwarf satellites was protracted for longer than $1 \mathrm{Gyr}$, as argued in Grebel and Gallagher III [99] to prove that star formation in dwarf spheroidals is not stopped by reionization feedback. 


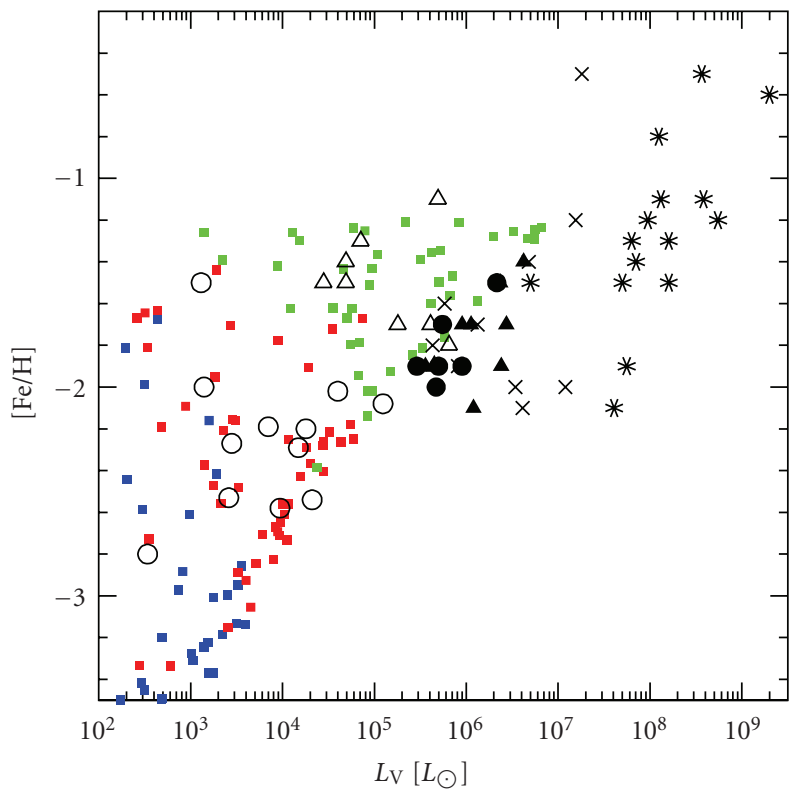

(a)

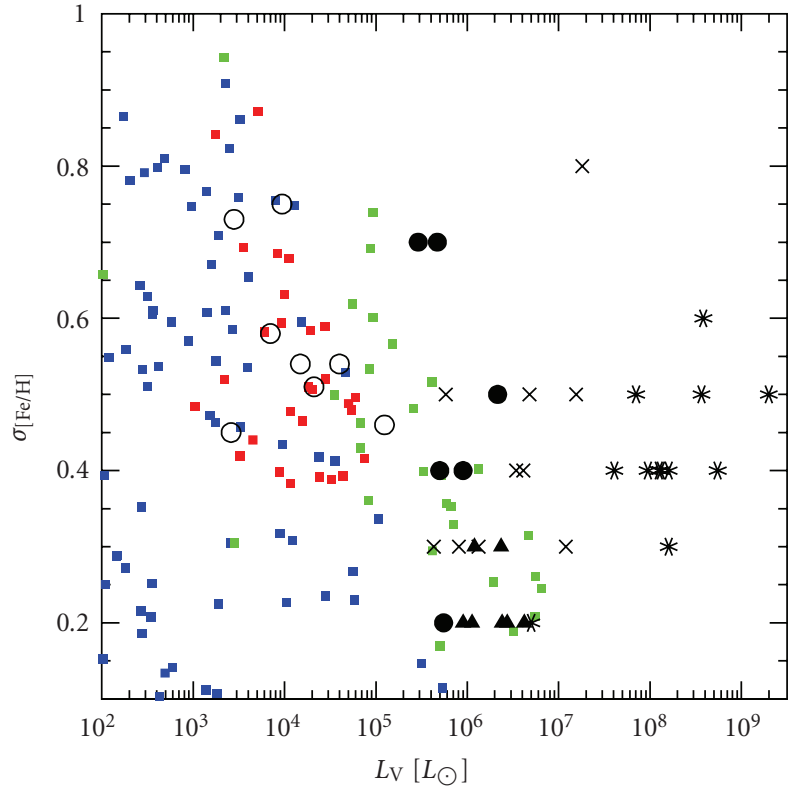

(b)

Figure 11: (a) Mean metallicity of the stars versus. V-band luminosity for Local Group dSphs plotted against RG05 predictions. They symbols have the same meaning as in Figure 8. In this plot simulated fossils (shown as small squares) are color coded according to their star formation efficiency, $f_{*}$ : red symbols show simulated dwarfs with $f_{*}<0.003$, blue $0.003 \leq f_{*} \leq 0.03$ and green $f_{*}>0.03$. (b) Same as (a) but showing the spread of the stellar metallicities in each dwarf (i.e., variance of the metallicity distribution) versus. their V-band luminosity.

\section{Discussion: The Tidal Scenario versus Fossil Hypothesis}

According to the results summarized above in Table 1, the number of dark matter satellites of the Milky Way that have or had in the past $v_{\max }>20 \mathrm{~km} \mathrm{~s}^{-1}$ is smaller than the number of observed luminous satellites (after applying completeness corrections). This implies that nonfossil galaxies cannot account for all the observed satellites. Thus, prereionization fossils should exist.

However, we have already discussed the several uncertainties in estimating the numbers summarized in Table 1. Additional uncertainties that render the identification of fossils uncertain are the following. The mass and circular velocity of the dark matter halo of the Milky Way are not known precisely. Simulations should take into account the effect of baryons in modifying the density profile and the radial distribution of satellites. The effect of tidal stripping on the properties of the stars in the satellites is not well understood, thus we do not know if the tidal scenario is consistent with observations of ultrafaint dwarfs. The luminosity and stellar properties of nonfossil dwarf satellites are not known.

Nonfossil galaxies with $v_{\max }>20 \mathrm{~km} \mathrm{~s}^{-1}$ may lose a substantial fraction of their mass due to tidal interactions. If they survive the interaction, their properties, such as surface brightness and half light radius, may be modified. Kravtsov et al. [91] estimate that about 10\% of Milky Way dark matter satellites were at least ten times more massive at their formation than they are today. Although their simulation does not include stars, they favor the idea that the stellar properties of these halos would remain unchanged (i.e., $\mathrm{dSphs}$ are not tidally stripped dIrr). In their model the majority of brighter dwarf satellites have been considerably more massive in the past and could have formed their stars undisturbed after reionization. More precisely, the redshift of reionization does not affect the results of their model for classic dwarfs because the probability of these to be fossils is low.

This version of the tidal model may be hard to distinguish observationally from the model we propose for the fossils because in both models the properties of the dwarfs are not modified by tidal forces (i.e., their properties are those at formation). In addition, fossil dwarfs may stop forming stars for only about 2 to $4 \mathrm{Gyr}$ after reionization, before starting to accrete gas again from the IGM. Thus, reionization may imprint a bimodal star formation history in some fossils, but this signature is not a robust discriminant because the star formation history of dwarfs cannot be determined with sufficient accuracy.

Observations seem to suggest that star formation in dwarf galaxies slightly more massive than $10^{8}-10^{9} \mathrm{M}_{\odot}$ may be similar to star formation in fossils and thus fit the observed properties of classical $\mathrm{dSphs}$ without requiring significant tidal stripping of stars. If star formation was included in Kravtsov et al. [91], their model may have reproduced the properties of observed dwarf satellites that our simulations of pre-reionization dwarf galaxies already does. The differences between the two models will depend on whether fossil galaxies are allowed to form and on their properties. 
In other words, the two models may differ on the assumed mass of the smallest dark halo that can host luminous satellites. This critical mass cannot be directly observed in dwarf galaxies but, in principle, can be constrained by comparing the observed number of luminous satellites to the model predictions. Determining the minimum mass for a dark halo to become luminous is of great importance in understanding galaxy formation in the early universe.

To summarize, there are a few observational tests that can be used to distinguish true fossils from dSphs or dEs that form in more massive halos and form stars unaffected by IGM reionization. True fossils should have either a single old stellar population or have a bimodal star formation history produced by a temporary suppression of star formation after reionization and late gas accretion. In addition, if the number of observed Milky Way satellites (or the number of isolated dwarfs) exceeds some critical value determined using N-body simulations (e.g., see Table 1), we may conclude that some pre-reionization fossils do exist in the Local Group.

It is likely that these tests will prove inconclusive for some time to come, unless the number (after corrections for completeness) of new ultrafaint galaxies surges in the coming years. The weakness of the star formation history test is that it requires measurements with precision of 1-2 Gyr of the stellar populations in order to be really discriminating between models that are quite similar to each other. This is hard to achieve especially for ultrafaint dwarfs with few stars. If the number of ultrafaint dwarfs remains about the same as today, the number argument may also remain controversial until more detailed theoretical modeling can reduce the current uncertainties surrounding the expected number of dark halos in the Milky Way and the completeness corrections of the observations. Ultimately, the case for the origin of ultrafaint dwarfs must be made on the basis of the model that does the best job of reproducing available observations.

Finally, even if pre-reionization fossils do not exist (i.e., halos with $v_{\max }<20 \mathrm{~km} \mathrm{~s}^{-1}$ are all dark), a fraction of them should be able to accrete some gas at redshift $z<$ $1-2$ and might be discoverable in the outer parts of the Local Group using $\mathrm{H} \alpha$ or $21 \mathrm{~cm}$ surveys (e.g., ALFALFA survey $[100,101])$. Of course, one should prove that the gas clouds are embedded in dark halos. Measurements of the gas cloud size, column density, and velocity broadening of the emission/absorption lines can be used to discriminate between "dark galaxies" and tidal debris. This is because the gas in dark galaxies is confined by the gravitational potential of the dark matter halo, while tidal debris or clouds formed via thermal instability are confined by the external gas pressure [3]. This is another promising direction for determining the minimum mass of luminous galaxies in the universe.

Another variant of the tidal hypothesis for the origin of dSphs is a scenario in which dIrr galaxies transform into dSphs as they fall into the Milky Way and Andromeda, due to tidal and ram pressure stripping $[59,102]$. A work by Peñarrubia et al. [60] explores the idea that ultrafaint dSphs are tidally stripped dIrrs. They achieve some success in reproducing observed properties of ultrafaint dwarfs. While this type of tidal stripping can reproduce properties of an individual galaxy, it is unable to completely reproduce all the trends in the ultrafaint population. This is primarily seen in the kinematics of the ultrafaint dwarfs. Tidal stripping predicts a steeper than observed drop in the velocity dispersion of the stars with decreasing $L_{\mathrm{V}}$ [60]. In addition several dSph do not show signs of strong tidal stripping. And XII and And XIV may be on their first approach to the Local Group $[22,103]$. Other examples of dSphs that are found distant from the center of their host galaxies are And XVIII, Cetus and Tucana [104].

Finally, another interesting case study is Leo $\mathrm{T}$, that we have discussed extensively above in Sections 2.2 and 4 . Leo $\mathrm{T}$ properties can be explained in some detail as being a fossil that experienced a late phase of gas accretion [3]. However, another possibility that should be explored quantitatively with simulations is that Leo $\mathrm{T}$ is more massive than a fossil but less massive than dIrr galaxies.

\section{Conclusions and Future Work}

We have summarized our work on the formation of the first galaxies before reionization (i.e., pre-reionization dwarfs) and the quest to identify the fossils of these first galaxies in the Local Group. The definition of a pre-reionization fossil is not directly related to the suppression of star formation experienced by these galaxies due to reionization feedback. Indeed, we discussed how pre-reionization fossils may experience a late phase of gas accretion and possibly star formation at redshift $z<1-2$. Most importantly, fossils are a population of dwarf galaxies whose formation (i.e., the fraction of halos that are luminous) is self-regulated on cosmological distance scales by radiative processes. Their existence is not certain due to a possible strong negative feedback that may prevent the majority of these halos from ever forming stars. In addition, if negative feedback heavily suppresses the number and luminosity of these first galaxies, more massive halos with $v_{\max }>20 \mathrm{~km} \mathrm{~s}^{-1}$ will evolve differently because of the lower level of metal preenrichment of the IGM. To summarize, the critical circular velocity $v_{\max } \sim 20 \mathrm{~km} \mathrm{~s}^{-1}$ that we adopt to define a fossil is primarily motivated by fundamental differences in cooling and feedback processes that regulate star formation in these halos in the early universe. However, it is also close to the critical value for continued gas accretion after IGM reionization $[2,40,86]$.

The number of Milky Way and M31 satellites provides an indirect test of galaxy formation and the importance of positive and negative feedback in the early universe. This test, although the uncertainties are large, supports the idea that a fraction of the new ultrafaint dwarfs are fossils. The good agreement of the SDSS and new M31 ultrafaint dwarf properties with predictions of our simulations (RG05, GK06, [51]) does not prove the primordial origin of the new ultrafaint dwarfs, but it supports this possibility.

More theoretical work and more observational data are needed to prove that some dwarfs in the Local Group are true fossils of the first galaxies. Future theoretical work should focus on improving the accuracy of predictions on 
the properties of dwarf galaxies formed before reionization and their evolution to the present day. Modeling the evolution of the baryonic component after reionization in dwarf satellites and in the Milky Way-Andromeda system may be necessary to make robust predictions. More observational data will certainly be available in the near future. A large number of surveys, both at optical and radio wavelengths will be online in the near future (e.g., Pan-STARRS, LSST, ALMA, EVLA, JWST, SKA to mention a few). Different survey strategies may be used to find and characterize fossil dwarf galaxies. A deep pencil beam survey would be useful to find the faintest dwarf satellites of the Milky Way and determine more precisely their Galactocentric distribution. A willower all sky survey could be used to quantify the degree of anisotropy in the distribution of satellites around the Milky Way.

The star formation history of the dwarf galaxies is not strongly discriminatory because fossil galaxies may have a late phase of gas accretion and star formation during the last 9-10 Gyrs [3]. The distinction between fossils and nonfossil galaxies may be quite elusive but it is nevertheless important to understand galaxy formation and feedback in the early universe. Arguments based on counting the number of dwarfs in the Local universe are among the more solid arguments that could be used to prove the existence of fossil galaxies (see Table 1).

Future tests may be provided by deep surveys looking for ultrafaint galaxies in the local voids or looking for gas in dark galaxies (i.e., dark halos that have been able to accrete gas from the IGM at $z<1-2$ ). Ultra-faint dwarfs should be present in the voids if dwarf galaxies formed in large numbers before reionization (Bovill \& Ricotti, in preparation). If prereionization dwarfs never formed due to dominant negative feedback in the early universe, it is possible that a faint (in $\mathrm{H} \alpha$ and $21 \mathrm{~cm}$ emission) population of dark galaxies exists in the outer parts of the Local Group. Hence, another way to detect fossil galaxies in the outer parts of the Milky Way or outside the super-galactic plane would be to search for neutral or ionized gas that they may have accreted from the IGM. Future radio telescopes (e.g., ALMA, EVLA, SKA) may be able to detect neutral hydrogen in dark galaxies or in ultrafaint dwarfs. Ionized gas in the outer parts of dark halos may be observed in absorption along the line of sight of distant quasars (e.g., in O VI or O IV with COS on the HST). However, the probability that a line of sight toward a quasar intersects the ionized gas collected from the IGM by dark or fossil galaxies might be small. Additional theoretical work is required to address these issues.

\section{References}

[1] N. Y. Gnedin and J. P. Ostriker, "Reionization of the universe and the early production of metals," The Astrophysical Journal, vol. 486, no. 2, pp. 581-598, 1997.

[2] M. Hoeft, G. Yepes, S. Gottlöber, and V. Springel, "Dwarf galaxies in voids: suppressing star formation with photoheating," Monthly Notices of the Royal Astronomical Society, vol. 371, no. 1, pp. 401-414, 2006.

[3] M. Ricotti, "Late gas accretion on to primordial minihaloes: a model for Leo T, dark galaxies and extragalactic high-velocity clouds," Monthly Notices of the Royal Astronomical Society, vol. 392, pp. L45-L49, 2009.

[4] A. Klypin, A. V. Kravtsov, O. Valenzuela, and F. Prada, "Where are the missing galactic satellites?" The Astrophysical Journal, vol. 522, no. 1, pp. 82-92, 1999.

[5] B. Moore, T. Quinn, F. Governato, J. Stadel, and G. Lake, "Cold collapse and the core catastrophe," Monthly Notices of the Royal Astronomical Society, vol. 310, no. 4, pp. 1147-1152, 1999.

[6] I. D. Karachentsev, V. E. Karachentseva, W. K. Huchtmeier, and D. I. Makarov, "A catalog of neighboring galaxies," The Astronomical Journal, vol. 127, no. 4, pp. 2031-2068, 2004.

[7] I. D. Karachentsev, A. Dolphin, R. B. Tully, et al., "Advanced camera for surveys imaging of 25 galaxies in nearby groups and in the field," The Astronomical Journal, vol. 131, no. 3, pp. 1361-1376, 2006.

[8] R. B. Tully, L. Rizzi, A. E. Dolphin, et al., "Associations of dwarf galaxies," Astronomical Journal, vol. 132, no. 2, pp. 729748, 2006.

[9] J. L. Tinker and C. Conroy, "The void phenomenon explained," The Astrophysical Journal, vol. 691, pp. 633-639, 2009.

[10] P. Bode, J. P. Ostriker, and N. Turok, "Halo formation in warm dark matter models," The Astrophysical Journal, vol. 556, no. 1, pp. 93-107, 2001.

[11] Z. Haiman, T. Abel, and M. J. Rees, "The radiative feedback of the first cosmological objects," The Astrophysical Journal, vol. 534, no. 1, pp. 11-24, 2000.

[12] V. Belokurov, D. B. Zucker, N. W. Evans, et al., "A faint new Milky Way satellite in bootes," The Astrophysical Journal, vol. 647, no. 2, pp. L111-L114, 2006.

[13] V. Belokurov, D. B. Zucker, N. W. Evans, et al., "Cats and dogs, hair and a hero: a quintet of new Milky Way companions," The Astrophysical Journal, vol. 654, no. 2, pp. 897-906, 2007.

[14] M. J. Irwin, V. Belokurov, N. W. Evans, et al., "Discovery of an unusual dwarf galaxy in the outskirts of the Milky Way," The Astrophysical Journal, vol. 656, no. 1, pp. L13-L16, 2007.

[15] S. M. Walsh, H. Jerjen, and B. Willman, "A pair of boötes: a new Milky Way satellite," The Astrophysical Journal, vol. 662, no. 2, pp. L83-L86, 2007.

[16] B. Willman, J. J. Dalcanton, D. Martinez-Delgado, et al., "A new Milky Way dwarf galaxy in URSA major," The Astrophysical Journal, vol. 626, no. 2, pp. L85-L88, 2005.

[17] B. Willman, M. R. Blanton, A. A. West, et al., "A new Milky Way companion: Unusual globular cluster or extreme dwarf satellite?" The Astronomical Journal, vol. 129, no. 6, pp. 26922700, 2005.

[18] D. B. Zucker, V. Belokurov, N. W. Evans, et al., "A new Milky Way dwarf satellite in canes venatici," The Astrophysical Journal, vol. 643, no. 2, pp. L103-L106, 2006.

[19] D. B. Zucker, V. Belokurov, N. W. Evans, et al., "A curious Milky Way satellite in Ursa Major," The Astrophysical Journal, vol. 650, no. 1, pp. L41-L44, 2006.

[20] R. Ibata, N. F. Martin, M. Irwin, et al., "The haunted halos of andromeda and triangulumml: a panorama of galaxy formation in action," The Astrophysical Journal, vol. 671, no. 2, pp. 1591-1623, 2007.

[21] S. R. Majewski, R. L. Beaton, R. J. Patterson, et al., "Discovery of andromeda XIV: a dwarf spheroidal dynamical rogue in the local group?" The Astrophysical Journal, vol. 670, no. 1, pp. L9-L12, 2007.

[22] N. F. Martin, R. A. Ibata, M. J. Irwin, et al., "Discovery and analysis of three faint dwarf galaxies and a globular cluster in 
the outer halo of the Andromeda galaxy," Monthly Notices of the Royal Astronomical Society, vol. 371, no. 4, pp. 1983-1991, 2006.

[23] J. D. Simon and M. Geha, "The kinematics of the ultra-faint Milky Way satellites: solving the missing satellite problem," The Astrophysical Journal, vol. 670, no. 1, pp. 313-331, 2007.

[24] S. Koposov, V. Belokurov, N. W. Evans, et al., "The luminosity function of the Milky Way satellites," The Astrophysical Journal, vol. 686, no. 1, pp. 279-291, 2008.

[25] E. J. Tollerud, J. S. Bullock, L. E. Strigari, and B. Willman, "Hundreds of Milky Way satellites? Luminosity bias in the satellite luminosity function," The Astrophysical Journal, vol. 688, no. 1, pp. 277-289, 2008.

[26] M. Geha, B. Willman, J. D. Simon, et al., "The least-luminous galaxy: spectroscopy of the Milky Way satellite segue 1," The Astrophysical Journal, vol. 692, no. 2, pp. 1464-1475, 2009.

[27] A. Babul and M. J. Rees, "On dwarf elliptical galaxies and the faint blue counts," Monthly Notices of the Royal Astronomical Society, vol. 255, pp. 346-350, 1992.

[28] G. Efstathiou, "Suppressing the formation of dwarf galaxies via photoionization," Monthly Notices of the Royal Astronomical Society, vol. 256, no. 2, pp. 43P-47P, 1992.

[29] P. R. Shapiro, M. L. Giroux, and A. Babul, "Reionization in a cold dark matter universe: the feedback of galaxy formation on the intergalactic medium," The Astrophysical Journal, vol. 427, no. 1, pp. 25-50, 1994.

[30] Z. Haiman, M. J. Rees, and A. Loeb, " $\mathrm{H}_{2}$ cooling of primordial gas triggered by UV irradiation," The Astrophysical Journal, vol. 467, no. 2, pp. 522-531, 1996.

[31] A. A. Thoul and D. H. Weinberg, "Hydrodynamic simulations of galaxy formation. II. Photoionization and the formation of low-mass galaxies," The Astrophysical Journal, vol. 465, no. 2, pp. 608-616, 1996.

[32] T. Quinn, N. Katz, and G. Efstathiou, "Photoionization and the formation of dwarf galaxies," Monthly Notices of the Royal Astronomical Society, vol. 278, no. 4, pp. L49-L54, 1996.

[33] D. H. Weinberg, L. Hernquist, and N. Katz, "Photoionization, numerical resolution, and galaxy formation," The Astrophysical Journal, vol. 477, no. 1, pp. 8-20, 1997.

[34] J. F. Navarro and M. Steinmetz, "The effects of a photoionizing ultraviolet background on the formation of disk galaxies," The Astrophysical Journal, vol. 478, no. 1, pp. 13-28, 1997.

[35] J. S. Bullock, A. V. Kravtsov, and D. H. Weinberg, "Reionization and the abundance of galactic satellites," The Astrophysical Journal, vol. 539, no. 2, pp. 517-521, 2000.

[36] N. Y. Gnedin, "Cosmological reionization by stellar sources," The Astrophysical Journal, vol. 535, no. 2, pp. 530-554, 2000.

[37] R.S. Somerville, "Can photoionization squelching resolve the substructure crisis?” The Astrophysical Journal, vol. 572, no. 1, pp. L23-L26, 2002.

[38] M. Dijkstra, Z. Haiman, and A. Loeb, "A limit from the X-ray background on the contribution of quasars to reionization," The Astrophysical Journal, vol. 613, no. 2, pp. 646-654, 2004.

[39] P. R. Shapiro, I. T. Iliev, and A. C. Raga, "Photoevaporation of cosmological minihaloes during reionization," Monthly Notices of the Royal Astronomical Society, vol. 348, no. 3, pp. 753-782, 2004.

[40] T. Okamoto, L. Gao, and T. Theuns, "Mass loss of galaxies due to an ultraviolet background," Monthly Notices of the Royal Astronomical Society, vol. 390, no. 3, pp. 920-928, 2008.

[41] M. Ricotti, N. Y. Gnedin, and J. M. Shull, "The fate of the first galaxies. II. Effects of radiative feedback," The Astrophysical Journal, vol. 575, no. 1, pp. 49-67, 2002.
[42] J. H. Wise and T. Abel, "Resolving the formation of protogalaxies. III. Feedback from the first stars," The Astrophysical Journal, vol. 685, no. 1, pp. 40-56, 2008.

[43] B. W. O'Shea and M. L. Norman, "Population III star formation in a $\Lambda \mathrm{CDM}$ universe. II. Effects of a photodissociating background," The Astrophysical Journal, vol. 673, no. 1, pp. 14-33, 2008.

[44] M. Ricotti, N. Y. Gnedin, and J. M. Shull, "The fate of the first galaxies. III. Properties of primordial dwarf galaxies and their impact on the intergalactic medium," The Astrophysical Journal, vol. 685, no. 1, pp. 21-39, 2008.

[45] J. H. Wise and T. Abel, "Suppression of $\mathrm{H}_{2}$ cooling in the ultraviolet background," The Astrophysical Journal, vol. 671, no. 2, pp. 1559-1567, 2007.

[46] M. E. Machacek, G. L. Bryan, and T. Abel, "Simulations of pregalactic structure formation with radiative feedback," The Astrophysical Journal, vol. 548, no. 2, pp. 509-521, 2001.

[47] M. E. Machacek, G. L. Bryan, and T. Abel, "Effects of a soft X-ray background on structure formation at high redshift," Monthly Notices of the Royal Astronomical Society, vol. 338, no. 2, pp. 273-286, 2003.

[48] M. Ricotti, N. Y. Gnedin, and J. M. Shull, "The fate of the first galaxies. I. Self-consistent cosmological simulations with radiative transfer," The Astrophysical Journal, vol. 575, no. 1, pp. 33-48, 2002.

[49] M. Ricotti, N. Y. Gnedin, and J. M. Shull, "Feedback from galaxy formation: production and photodissociation of primordial $\mathrm{H}_{2}$," The Astrophysical Journal, vol. 560, no. 2, pp. 580-591, 2001.

[50] M. Ricotti and N. Y. Gnedin, "Formation histories of dwarf galaxies in the local group," The Astrophysical Journal, vol. 629, no. 1, pp. 259-267, 2005.

[51] M. S. Bovill and M. Ricotti, "Pre-reionization fossils, ultrafaint dwarfs, and the missing galactic satellite problem," The Astrophysical Journal, vol. 693, pp. 1859-1870, 2009.

[52] V. Bromm, A. Ferrara, P. S. Coppi, and R. B. Larson, "The fragmentation of pre-enriched primordial objects," Monthly Notices of the Royal Astronomical Society, vol. 328, no. 3, pp. 969-976, 2001.

[53] F. Santoro and J. M. Shuli, "Critical metallicity and finestructure emission of primordial gas enriched by the first stars," The Astrophysical Journal, vol. 643, no. 1, pp. 26-37, 2006.

[54] B. D. Smith, M. J. Turk, S. Sigurdsson, B. W. O'Shea, and M. L. Norman, "Three modes of metal-enriched star formation in the early universe," The Astrophysical Journal, vol. 691, pp. 441-451, 2009.

[55] S. Salvadori and A. Ferrara, "Ultra faint dwarfs: probing early cosmic star formation," Monthly Notices of the Royal Astronomical Society, vol. 395, pp. L6-L10, 2009.

[56] M. Ricotti, N. Y. Gnedin, and J. M. Shull, "The evolution of the effective equation of state of the intergalactic medium," The Astrophysical Journal, vol. 534, no. 1, pp. 41-56, 2000.

[57] S. E. Koposov, J. Yoo, H.-W. Rix, D. H. Weinberg, A. V. Macciò, and J. M. Escudé, "A quantitative explanation of the observed population of Milky Way satellite galaxies," The Astrophysical Journal, vol. 696, pp. 2179-2194, 2009.

[58] M. Mateo, "Dwarf galaxies of the local group," Annual Review of Astronomy and Astrophysics, vol. 36, no. 1, pp. 435-506, 1998.

[59] L. Mayer, S. Kazantzidis, C. Mastropietro, and J. Wadsley, "Early gas stripping as the origin of the darkest galaxies in the universe," Nature, vol. 445, no. 7129, pp. 738-740, 2007. 
[60] J. Peñarrubia, J. F. Navarro, and A. W. McConnachie, "The tidal evolution of local group dwarf spheroidals," The Astrophysical Journal, vol. 673, no. 1, pp. 226-240, 2008.

[61] S. M. Walsh, B. Willman, and H. Jerjen, "The invisibles: a detection algorithm to trace the faintest Milky Way satellites," The Astronomical Journal, vol. 137, no. 1, pp. 450-469, 2009.

[62] N. F. Martin, A. W. McConnachie, M. Irwin, L. M. Widrow, A. M.N. Ferguson, R. A. Ibata, J. Dubinski, A. Babul, S. Chapman, M. Fardal, G. F. Lewis, J. Navarro, and R. M. Rich, "PandAS' Cubs: discovery of two new dwarf galaxies in the surroundings of the Andromeda and triangulum galaxies," Astrophysical Journal, vol. 705, no. 1, pp. 758-765, 2009.

[63] J. K. Adelman-McCarthy, M. A. Agüeros, S. S. Allam, et al., "The fourth data release of the sloan digital sky survey," The Astrophysical Journal, Supplement Series, vol. 162, no. 1, pp. 38-48, 2006.

[64] J. K. Adelman-McCarthy, M. A. Agüeros, S. S. Allam, et al., "The fifth data release of the sloan digital sky survey," The Astrophysical Journal, Supplement Series, vol. 172, no. 2, pp. 634-644, 2007.

[65] P. Kroupa, C. Theis, and C. M. Boily, "The great disk of Milky-Way satellites and cosmological sub-structures," Astronomy \& Astrophysics, vol. 431, no. 2, pp. 517-521, 2005.

[66] A. R. Zentner, A. V. Kravtsov, O. Y. Gnedin, and A. A. Klypin, "The anisotropic distribution of galactic satellites," The Astrophysical Journal, vol. 629, no. 1, pp. 219-232, 2005.

[67] N. F. Martin, M. G. Coleman, J. T. A. de Jong, et al., "A deep large binocular telescope view of the Canes Venatici I dwarf galaxy," The Astrophysical Journal, vol. 672, no. 1, pp. L13L16, 2008.

[68] J. T. A. de Jong, J. Harris, M. G. Coleman, et al., "The structural properties and star formation history of Leo $\mathrm{T}$ from deep LBT photometry," The Astrophysical Journal, vol. 680, no. 2, pp. 1112-1119, 2008.

[69] N. F. Martin, J. T. A. de Jong, and H.-W. Rix, "A comprehensive maximum likelihood analysis of the structural properties of faint Milky Way satellites," The Astrophysical Journal, vol. 684, no. 2, pp. 1075-1092, 2008.

[70] V. Bromm, P. S. Coppi, and R. B. Larson, "The formation of the first stars-I: the primordial star-forming cloud," The Astrophysical Journal, vol. 564, no. 1, pp. 23-51, 2002.

[71] T. Abel, G. L. Bryan, and M. L. Norman, "The formation of the first star in the universe," Science, vol. 295, no. 5552, pp. 93-98, 2002.

[72] K. Saigo, T. Matsumoto, and M. Umemura, "The formation of population III binaries," The Astrophysical Journal, vol. 615, no. 2, pp. L65-L68, 2004.

[73] L. Gao, S. D. M. White, A. Jenkins, C. S. Frenk, and V. Springel, "Early structure in $\Lambda \mathrm{CDM}$, , Monthly Notices of the Royal Astronomical Society, vol. 363, no. 2, pp. 379-392, 2005.

[74] B. W. O'Shea and M. L. Norman, "Population III star formation in a ACDM universe. I. The effect of formation redshift and environment on protostellar accretion rate," The Astrophysical Journal, vol. 654, no. 1, pp. 66-92, 2007.

[75] N. Yoshida, K. Omukai, and L. Hernquist, "Protostar formation in the early universe," Science, vol. 321, no. 5889, pp. 669-671, 2008.

[76] M. J. Turk, T. Abel, and B. O'Shea, "The formation of population III binaries from cosmological initial conditions," Science, vol. 325, no. 5940, pp. 601-605, 2009.

[77] A. Stacy, T. H. Greif, and V. Bromm, "The first stars: formation of binaries and small multiple systems," Monthly Notices of the Royal Astronomical Society, 12 pages, January 2010.
[78] B. Ciardi, A. Ferrara, and T. Abel, "Intergalactic $\mathrm{H}_{2}$ photodissociation and the soft ultraviolet background produced by population III objects," The Astrophysical Journal, vol. 533, no. 2, pp. 594-600, 2000.

[79] M. E. Machacek, G. L. Bryan, A. Meiksin, et al., "Hydrodynamical simulations of the Ly $\alpha$ forest: model comparisons," The Astrophysical Journal, vol. 532, no. 1, pp. 118-135, 2000.

[80] D. Whalen, B. W. O'Shea, J. Smidt, and M. L. Norman, "Photoionization of clustered halos by the first stars," in First Stars III, vol. 990 of AIP Conference Proceedings, pp. 381-385, Santa Fe, NM, USA, 2007.

[81] P. R. Shapiro and H. Kang, "Hydrogen molecules and the radiative cooling of pregalactic shocks," The Astrophysical Journal, vol. 318, pp. 32-65, 1987.

[82] M. A. Alvarez, V. Bromm, and P. R. Shapiro, "The H II region of the first star," The Astrophysical Journal, vol. 639, no. 2, pp. 621-632, 2006.

[83] B. Ciardi, E. Scannapieco, F. Stoehr, A. Ferrara, I. T. Iliev, and P. R. Shapiro, "The effect of minihaloes on cosmic reionization," Monthly Notices of the Royal Astronomical Society, vol. 366, no. 2, pp. 689-696, 2006.

[84] N. Y. Gnedin and T. Abel, "Multi-dimensional cosmological radiative transfer with a variable Eddington tensor formalism," New Astronomy, vol. 6, no. 7, pp. 437-455, 2001.

[85] C. C. Steidel, K. L. Adelberger, M. Giavalisco, M. Dickinson, and M. Pettini, "Lyman-break galaxies at $z>4$ and the evolution of the ultraviolet luminosity density at high redshift," Astrophysical Journal, vol. 519, no. 1, pp. 1-17, 1999.

[86] N. Y. Gnedin, "Effect of reionization on structure formation in the universe," The Astrophysical Journal, vol. 542, no. 2, pp. 535-541, 2000.

[87] J. S. Bullock, T. S. Kolatt, Y. Sigad, et al., "Profiles of dark haloes: evolution, scatter and environment," Monthly Notices of the Royal Astronomical Society, vol. 321, no. 3, pp. 559-575, 2001.

[88] R. H. Wechsler, J. S. Bullock, J. R. Primack, A. V. Kravtsov, and A. Dekel, "Concentrations of dark halos from their assembly histories," The Astrophysical Journal, vol. 568, no. 1, pp. 52-70, 2002.

[89] E. Bertschinger, "Self-similar secondary infall and accretion in an Einstein-de Sitter universe," The Astrophysical Journal, Supplement Series, vol. 58, p. 39, 1985.

[90] G. S. Stinson, J. J. Dalcanton, T. Quinn, T. Kaufmann, and J. Wadsley, "Breathing in low-mass galaxies: a study of episodic star formation," The Astrophysical Journal, vol. 667, no. 1, pp. 170-175, 2007.

[91] A. V. Kravtsov, O. Y. Gnedin, and A. A. Klypin, "The tumultuous lives of galactic dwarfs and the missing satellites problem," The Astrophysical Journal, vol. 609, no. 2, pp. 482497, 2004.

[92] V. Springel, J. Wang, M. Vogelsberger, et al., "The Aquarius Project: the subhaloes of galactic haloes," Monthly Notices of the Royal Astronomical Society, vol. 391, no. 4, pp. 1685-1711, 2008.

[93] J. Diemand, M. Kuhlen, and P. Madau, "Formation and evolution of galaxy dark matter halos and their substructure," The Astrophysical Journal, vol. 667, no. 2, pp. 859-877, 2007.

[94] N. Y. Gnedin and A. V. Kravtsov, "Fossils of reionization in the local group," The Astrophysical Journal, vol. 645, no. 2, pp. 1054-1061, 2006.

[95] J. Kormendy and K. C. Freeman, "Scaling laws for dark matter halos in late-type and dwarf spheroidal galaxies," in 
Proceedings of the IAU Symposium, p. 377, San Francisco, Calif, USA, 2004.

[96] N. F. Martin, R. A. Ibata, S. C. Chapman, M. Irwin, and G. F. Lewis, "A Keck/DEIMOS spectroscopic survey of faint Galactic satellites: searching for the least massive dwarf galaxies," Monthly Notices of the Royal Astronomical Society, vol. 380, no. 1, pp. 281-300, 2007.

[97] L. E. Strigari, J. S. Bullock, M. Kaplinghat, et al., "A common mass scale for satellite galaxies of the Milky Way," Nature, vol. 454, no. 7208, pp. 1096-1097, 2008.

[98] E. N. Kirby, J. D. Simon, M. Geha, P. Guhathakurta, and A. Frebel, "Uncovering extremely metal-poor stars in the Milky Way's ultrafaint dwarf spheroidal satellite galaxies," The Astrophysical Journal, vol. 685, no. 1, pp. L43-L46, 2008.

[99] E. K. Grebel and J. S. Gallagher III, "The impact of reionization on the stellar populations of nearby dwarf galaxies," The Astrophysical Journal, vol. 610, no. 2, pp. L89L92, 2004.

[100] R. Giovanelli, M. P. Haynes, B. R. Kent, et al., "The arecibo legacy fast ALFA survey. I. Science goals, survey design, and strategy," The Astronomical Journal, vol. 130, no. 6, pp. 25982612, 2005.

[101] R. Giovanelli, M. P. Haynes, B. R. Kent, et al., "The arecibo legacy fast alfa survey. III. H I source catalog of the northern virgo cluster region," The Astronomical Journal, vol. 133, no. 6, pp. 2569-2583, 2007.

[102] L. Mayer, C. Mastropietro, J. Wadsley, J. Stadel, and B. Moore, "Simultaneous ram pressure and tidal stripping; how dwarf spheroidals lost their gas," Monthly Notices of the Royal Astronomical Society, vol. 369, no. 3, pp. 1021-1038, 2006.

[103] S. C. Chapman, J. Peñarrubia, R. Ibata, et al., "Strangers in the night: discovery of a dwarf spheroidal galaxy on its first local group infall," The Astrophysical Journal, vol. 662, no. 2, pp. L78-L82, 2007.

[104] A. W. McConnachie, A. Huxor, N. F. Martin, et al., "A trio of new local group galaxies with extreme properties," Astrophysical Journal, vol. 688, no. 2, pp. 1009-1020, 2008. 

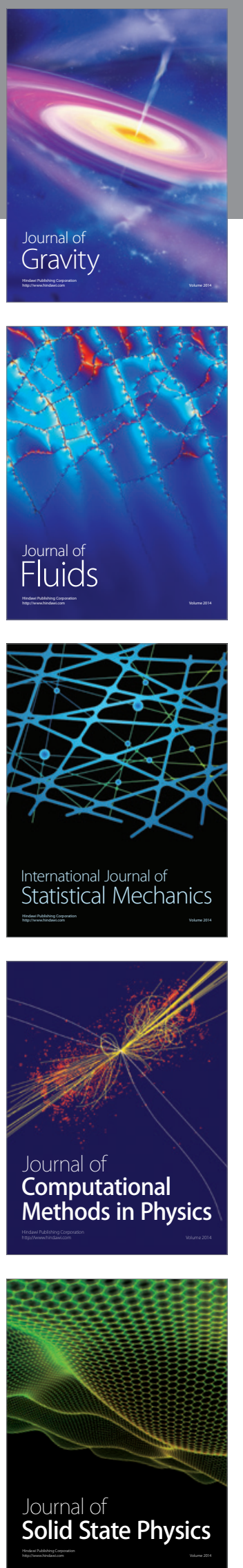

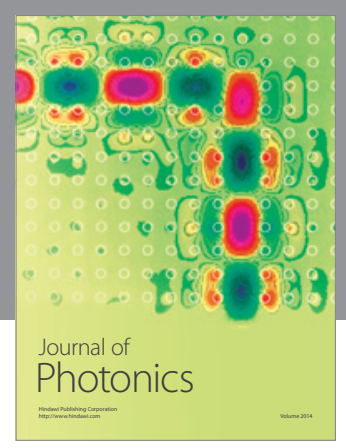

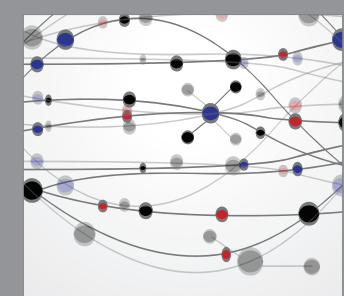

The Scientific World Journal
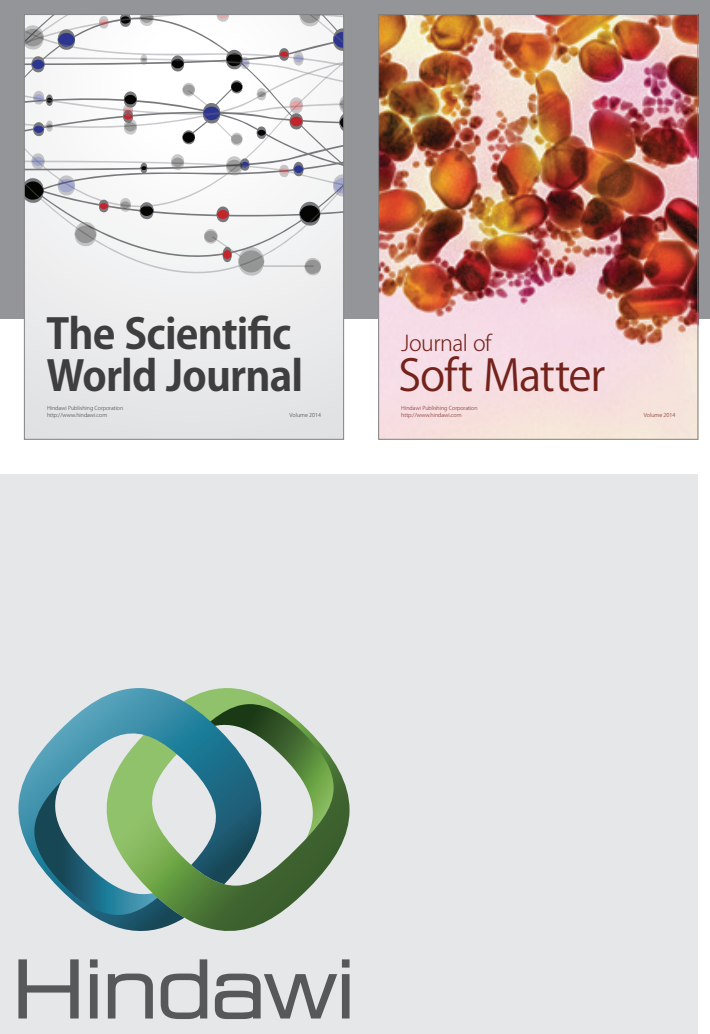

Submit your manuscripts at

http://www.hindawi.com
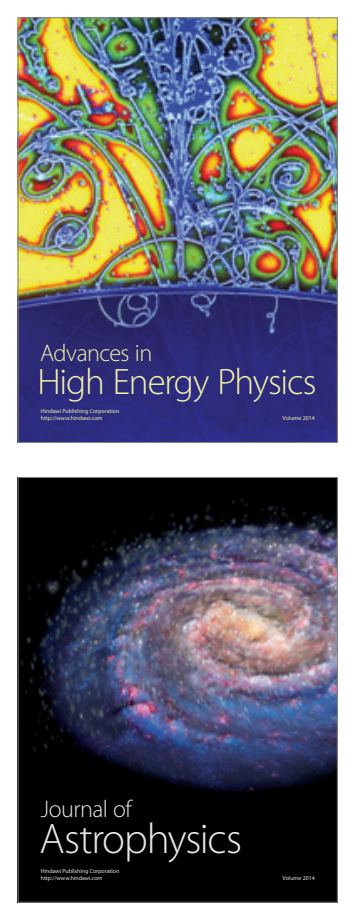
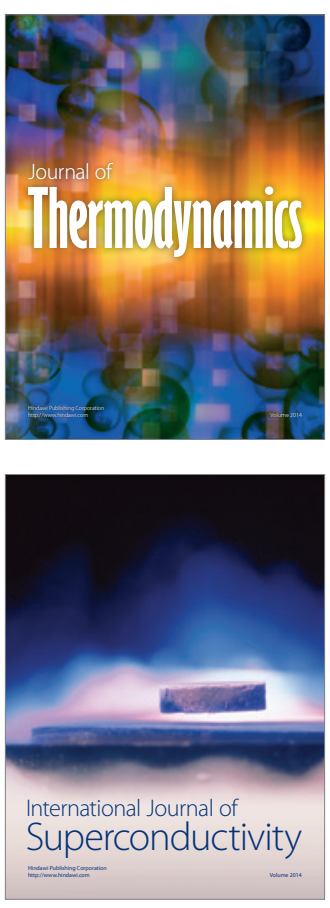
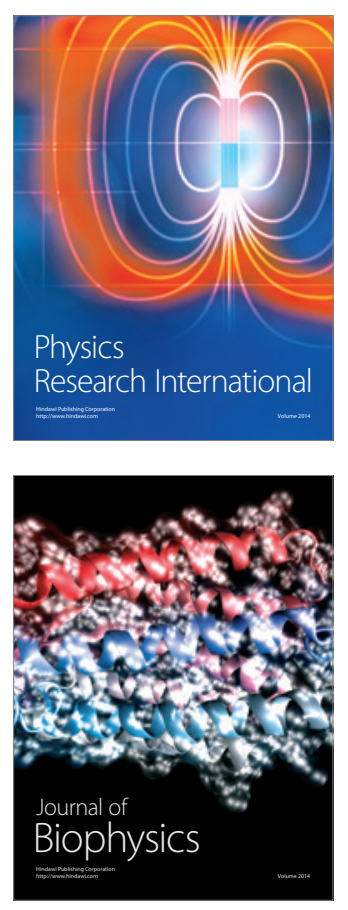
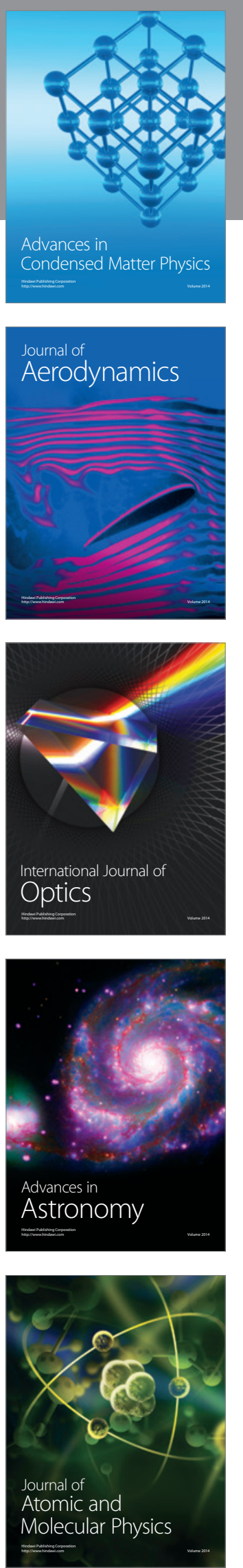